\title{
MOJAVE: MONITORING OF JETS IN ACTIVE GALACTIC NUCLEI WITH VLBA EXPERIMENTS. VI. KINEMATICS ANALYSIS OF A COMPLETE SAMPLE OF BLAZAR JETS
}

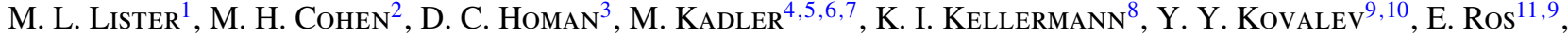 \\ T. SaVolainen ${ }^{9}$, And J. A. Zensus ${ }^{9}$ \\ ${ }^{1}$ Department of Physics, Purdue University, 525 Northwestern Avenue, West Lafayette, IN 47907, USA; mlister@purdue.edu \\ ${ }^{2}$ Department of Astronomy, California Institute of Technology, Mail Stop 249-17, Pasadena, CA 91125, USA; mhc@ astro.caltech.edu \\ ${ }^{3}$ Department of Physics and Astronomy, Denison University, Granville, OH 43023, USA; homand@ denison.edu \\ ${ }^{4}$ Dr. Remeis-Sternwarte Bamberg, Universität Erlangen-Nürnberg, Sternwartstrasse 7, 96049 Bamberg, Germany; matthias.kadler@ sternwarte.uni-erlangen.de \\ ${ }^{5}$ Erlangen Centre for Astroparticle Physics, Erwin-Rommel Str. 1, 91058 Erlangen, Germany \\ ${ }^{6}$ CRESST/NASA Goddard Space Flight Center, Greenbelt, MD 20771, USA \\ ${ }^{7}$ Universities Space Research Association, 10211 Wincopin Circle, Suite 500 Columbia, MD 21044, USA \\ ${ }^{8}$ National Radio Astronomy Observatory, 520 Edgemont Road, Charlottesville, VA 22903-2475, USA; kkellerm@nrao.edu \\ ${ }^{9}$ Max-Planck-Institut für Radioastronomie, Auf dem Hügel 69, D-53121 Bonn, Germany; ykovalev@ mpifr-bonn.mpg.de, tsavolainen@ mpifr-bonn.mpg.de, \\ azensus@mpifr-bonn.mpg.de \\ ${ }^{10}$ Astro Space Center of Lebedev Physical Institute, Profsoyuznaya 84/32, 117997 Moscow, Russia \\ ${ }^{11}$ Departament d'Astronomia i Astrofísica, Universitat de València, E-46100 Burjassot, València, Spain; Eduardo.Ros@uv.es \\ Received 2009 July 28; accepted 2009 September 27; published 2009 November 5
}

\begin{abstract}
We discuss the jet kinematics of a complete flux-density-limited sample of 135 radio-loud active galactic nuclei (AGNs) resulting from a 13 year program to investigate the structure and evolution of parsec-scale jet phenomena. Our analysis is based on new $2 \mathrm{~cm}$ Very Long Baseline Array (VLBA) images obtained between 2002 and 2007, but includes our previously published observations made at the same wavelength, and is supplemented by VLBA archive data. In all, we have used 2424 images spanning the years 1994-2007 to study and determine the motions of 526 separate jet features in 127 jets. The data quality and temporal coverage (a median of 15 epochs per source) of this complete AGN jet sample represent a significant advance over previous kinematics surveys. In all but five AGNs, the jets appear one-sided, most likely the result of differential Doppler boosting. In general, the observed motions are directed along the jet ridge line, outward from the optically thick core feature. We directly observe changes in speed and/or direction in one third of the well-sampled jet components in our survey. While there is some spread in the apparent speeds of separate features within an individual jet, the dispersion is about three times smaller than the overall dispersion of speeds among all jets. This supports the idea that there is a characteristic flow that describes each jet, which we have characterized by the fastest observed component speed. The observed maximum speed distribution is peaked at $\sim 10 c$, with a tail that extends out to $\sim 50 c$. This requires a distribution of intrinsic Lorentz factors in the parent population that range up to $\sim 50$. We also note the presence of some rare low-pattern speeds or even stationary features in otherwise rapidly flowing jets that may be the result of standing re-collimation shocks, and/or a complex geometry and highly favorable Doppler factor.
\end{abstract}

Key words: BL Lacertae objects: general - galaxies: active - galaxies: jets - radio continuum: galaxies - quasars: general - surveys

Online-only material: color figures, figure sets, machine-readable and VO tables

\section{INTRODUCTION}

This is the sixth paper in a series in which we report results of the multi-epoch Very Long Baseline Array (VLBA) MOJAVE program to investigate the parsec-scale jet kinematics of a complete flux-density-limited sample of 135 active galactic nuclei (AGNs) in the northern sky at $2 \mathrm{~cm}$. Lister \& Homan (2005, Paper I) have described the initial source sample selection and first VLBA polarization epoch results, and in subsequent papers we have discussed the circular polarization characteristics (Homan \& Lister 2006, Paper II), kiloparsec-scale jet structure (Cooper et al. 2007, Paper III), and parent luminosity function (LF; 2008, Paper IV) of the sample. In Paper V (Lister et al. 2009), we presented a large set of $2 \mathrm{~cm}$ VLBA images obtained over a 13 year period beginning in 1994. A primary goal of our study is a characterization of the long-term apparent jet motions seen in these radio-loud AGNs. In this paper, we discuss the kinematic properties of the 135 AGN jets in the flux-density-limited MOJAVE sample (Paper V) based on this unprecedented large VLBA data set. ${ }^{12}$ We reported preliminary findings regarding a correlation between jet speeds of the MOJAVE AGN and their Fermi $\gamma$-ray emission properties in previous papers (Lister et al. 2009; Kovalev et al. 2009). In subsequent papers we discuss the polarization evolution, accelerations, and jet bending properties of the sample.

The properties of a less well-defined sample of radio-loud AGNs covering the period from 1994 August to 2001 March and through 2006 September were discussed in earlier papers by Kellermann et al. (2004) and Cohen et al. (2007). In these papers, we reported apparent jet velocities typically between 0 and $15 c$, with a distribution extending to about $35 c$, and found that different features within individual jets appear to move with comparable velocity. In general, we found that lower-luminosity AGNs have slower jets than the strong blazars. We also noted the presence of apparently stationary jet features, as well as the presence of bends and twists where the observed motions do

12 The MOJAVE data archive is maintained at http://www.physics.purdue.edu/ MOJAVE. 
not back-trace to the base of the jet, but are instead aligned with the local jet direction (i.e., they are non-radial). We found little or no evidence for backward motion toward the jet origin, such as might be expected from random pattern speeds. However, detailed analysis was complicated by individual features that may break up or combine with others in the jet (e.g., Kadler et al. 2008).

Cohen et al. (2007) have interpreted the observed speed distributions in terms of the intrinsic bulk flow Lorentz factors and (unbeamed) synchrotron luminosities, and reported results roughly consistent with simple relativistic beaming models where the pattern velocity is comparable to the flow velocity. In a few cases, we found clear changes in the direction and/or speed of individual jet features as they propagate along the jet (e.g., Homan et al. 2003).

We chose a wavelength of $2 \mathrm{~cm}$ for our VLBA observations to optimize both resolution and sensitivity, and to minimize the impact of adverse weather conditions. Complementary radiojet kinematic studies have been reported at shorter wavelengths with better angular resolution but lower sensitivity (Jorstad et al. $2001,2005)$ or at longer wavelengths with better sensitivity to lower surface brightness features but poorer resolution (Britzen et al. 2007, 2008; Piner et al. 2007; M. Kadler et al. 2009, in preparation).

Jorstad et al. (2001) used the VLBA at $7 \mathrm{~mm}$ and $1.3 \mathrm{~cm}$ to obtain multi-epoch observations of $33 \gamma$-ray blazars between 1993 and 1997. Like Kellermann et al. (2004), they found that the jets of EGRET-detected $\gamma$-ray AGNs appear to be systematically faster than non $\gamma$-ray AGNs, and argued that superluminal jet component ejection times occur at the time of $\gamma$-ray outbursts. Also using the VLBA, Jorstad et al. (2005) reported total and polarized $7 \mathrm{~mm}$ VLBA images of 15 AGNs at 19 epochs between 1998 and 2001 and derived Doppler and Lorentz factors using estimates of intrinsic size estimated from the timescale of radio outbursts.

At $6 \mathrm{~cm}$, Britzen et al. (2007) used an ad-hoc array and the VLBA to observe 293 AGNs from the Caltech-Jodrell Flat-Spectrum Survey (Taylor et al. 1996), typically for three epochs spaced over the period 1990-2000. They reported on the distribution of velocities for 266 AGNs that had good quality multi-epoch data and also found that the most luminous sources tend to have the fastest apparent motions (Britzen et al. 2008).

Piner et al. (2007) used data from the Radio Reference Frame Image Database ${ }^{13}$ obtained with the VLBA and up to seven other antennas at $3.4 \mathrm{~cm}$ to investigate jet motions in 87 radio-bright AGNs during the period 1994-1998. Their overall apparent speed distribution was peaked at low values, and ranged up to $\sim 30 c$.

The combined $2 \mathrm{~cm}$ VLBA Survey/MOJAVE data set presented here and in Paper V represents a significant improvement over prior studies in terms of statistical completeness, temporal coverage (typically 15 epochs per source over 10 years), and data quality (typical image resolution $\lesssim 1$ mas and rms image noise $\lesssim 0.4 \mathrm{Jy} \mathrm{beam}^{-1}$ ). In this paper, we exploit the longer time range afforded by the newer observations as well as the use of extensive archival VLBA data to study the distribution of jet speeds in AGNs and to explore in more detail non-radial and accelerating jet flows as well as the emerging evidence for slow pattern speed features found in otherwise rapidly flowing jets. The overall layout is as follows. In Section 2, we discuss the observational set and our method of defin-

\footnotetext{
13 http://rorf.usno.navy.mil/
}

ing the individual jet features. In Section 3, we describe the jet kinematic properties of the sample, and we discuss general statistical trends in Section 4. We summarize our overall findings in Section 5. We adopt a cosmology with $\Omega_{m}=0.27, \Omega_{\Lambda}=0.73$, and $H_{o}=71 \mathrm{~km} \mathrm{~s}^{-1} \mathrm{Mpc}^{-1}$. We use the convention $S_{v} \propto v^{\alpha}$ for the spectral index. We refer to the radio sources throughout either by their B1950 nomenclature, or commonly used aliases.

\section{OBSERVATIONS AND DATA REDUCTION}

\subsection{Observational Data}

The data analyzed in this study were presented in Paper V, and consist of a set of $24242 \mathrm{~cm}$ VLBA observations of the complete flux-density-limited MOJAVE sample of 135 AGNs spanning from 1994 August 31 to 2007 September 6. Although the typical restoring beam of the naturally weighted images has dimensions of 1.1 by 0.6 mas, the positions of individual jet features can be determined to much higher accuracy, as we describe in Section 2.2. The temporal coverage varies between 5 and 89 VLBA epochs per source, with a median of 15 epochs. In scheduling our observations, attempts were made to sample those AGNs with the most rapid jet motions more frequently. Some sources had considerably more archival $2 \mathrm{~cm}$ VLBA data (not from our program) owing to widespread community interest or their popularity as calibrators.

The MOJAVE sample is selected on the basis of compact (2 cm VLBA) flux density, which favors AGNs that have strongly beamed, fast jets viewed at small angles to the line of sight (i.e., blazars). The flux density limit ( $1.5 \mathrm{Jy}$ for declinations $\geqslant 0^{\circ}$ and $2 \mathrm{Jy}$ for $-20^{\circ} \leqslant \delta<0^{\circ}$ ) was chosen to provide a sufficiently large number of quasars $(N=101)$, BL Lac objects $(N=22)$, and radio galaxies $(N=8)$ to investigate statistical trends in the overall sample and within the optical sub-classes. The redshift information on the sample is currently $93 \%$ complete; the missing sources are mainly BL Lacs with featureless spectra. At the present time, only four sources $(0446+113$, 0648-165, 1213-172, and 2021+317) lack published optical counterparts. The full list of MOJAVE sources and their general properties can be found in Table 1 of Paper V.

\subsection{Gaussian Model Fitting}

The majority of the jets in our sample have relatively simple radio morphologies that consist of a bright (presumably stationary) "core" and a faint jet that typically extends for a few milliarcseconds on one side of the core (Paper V). Given the modest dynamic range of a few thousand to one in our images, the jets can be typically modeled as a series of distinct Gaussian features. For the purposes of tracking these jet features across multiple epochs, we carried out our analysis in the interferometric $(u, v)$ plane by fitting the brightest features in each source with two-dimensional Gaussian components using the "model fit" task in Difmap (Shepherd 1997). This method offers advantages over fitting done in the image plane, as it uses the full resolution capability of the VLBA (Kovalev et al. 2005). However, components defined in this way do not necessarily correspond to real features in the image, but instead may merely be mathematical constructs needed to reproduce a complex brightness distribution. In our previous analysis (Kellermann et al. 2004), we examined each image for continuity in position, flux density, and dimensions and fit Gaussian components in the image plane to determine component positions, thus sacrificing effective resolution for 
Table 1

Fitted Jet Components

\begin{tabular}{|c|c|c|c|c|c|c|c|c|}
\hline $\begin{array}{c}\text { Source } \\
\text { (1) }\end{array}$ & $\begin{array}{l}\text { I.D. } \\
\text { (2) }\end{array}$ & $\begin{array}{c}\text { Epoch } \\
\text { (3) }\end{array}$ & $\begin{array}{c}I \\
(\mathrm{Jy}) \\
(4)\end{array}$ & $\begin{array}{c}r \\
\text { (mas) } \\
(5)\end{array}$ & $\begin{array}{c}\text { P.A. } \\
\text { (deg) } \\
(6)\end{array}$ & $\begin{array}{c}\text { Maj. } \\
\text { (mas) } \\
(7)\end{array}$ & $\begin{array}{c}\text { Ratio } \\
(8)\end{array}$ & $\begin{array}{c}\text { Maj. P.A. } \\
\text { (deg) } \\
(9)\end{array}$ \\
\hline \multirow[t]{15}{*}{$0003-066$} & 0 & $2001 \operatorname{Jan} 21$ & 1.577 & 0.04 & 193.8 & 0.95 & 0.11 & 7 \\
\hline & 1 & & 0.124 & 6.06 & 281.8 & 1.89 & 1.00 & $\ldots$ \\
\hline & 2 & & 0.156 & 1.18 & 286.6 & 0.48 & 1.00 & $\ldots$ \\
\hline & 7 & & 0.029 & 2.88 & 283.4 & $\ldots$ & $\ldots$ & $\ldots$ \\
\hline & 0 & 2001 Oct 31 & 1.280 & 0.02 & 217.5 & 0.87 & 0.11 & 9 \\
\hline & 1 & & 0.146 & 6.49 & 284.0 & 1.32 & 1.00 & $\ldots$ \\
\hline & 2 & & 0.125 & 1.27 & 292.6 & 0.49 & 1.00 & $\ldots$ \\
\hline & 0 & 2002 May 19 & 1.210 & 0.18 & 7.2 & 0.46 & 0.27 & 346 \\
\hline & 1 & & 0.173 & 6.53 & 279.7 & 1.18 & 1.00 & $\ldots$ \\
\hline & 2 & & 0.057 & 1.51 & 289.7 & 0.64 & 1.00 & $\ldots$ \\
\hline & 3 & & 0.059 & 1.06 & 258.5 & 0.33 & 1.00 & $\ldots$ \\
\hline & 4 & & 0.619 & 0.64 & 190.2 & $\ldots$ & $\ldots$ & $\ldots$ \\
\hline & 7 & & 0.072 & 3.15 & 284.8 & 2.00 & 1.00 & $\ldots$ \\
\hline & 0 & 2003 Feb 5 & 2.349 & 0.05 & 204.7 & 0.89 & 0.25 & 2 \\
\hline & 1 & & 0.204 & 6.65 & 281.0 & 1.25 & 1.00 & $\ldots$ \\
\hline
\end{tabular}

Notes. Columns are as follows: (1) IAU Name (B1950.0), (2) component name (zero indicates core component), (3) observation epoch, (4) flux density in Jy, (5) position offset from the core component (or map center for the core component entries), (6) position angle with respect to the core component (or map center for the core component entries), (7) FWHM major axis of fitted Gaussian (milliacseconds), (8) axial ratio of fitted Gaussian, (9) major axis position angle of fitted Gaussian.

a Individual component epoch not used in kinematic fits.

(This table is available in its entirety in machine-readable and Virtual Observatory (VO) forms in the online journal. A portion is shown here for guidance regarding its form and content.)

robust solutions. As discussed in Section 2.2.2, in order to ensure against non-physical solutions in our kinematic analysis, as might be introduced by $(u, v)$ plane fitting, we have used only "robust" components with reliable identification across at least five epochs. Our fits yielded positions, sizes, and flux densities for distinct features in the jets, which are listed in Table 1.

An important constraint on our kinematic analysis is to be able to reliably cross-identify individual jet features from one epoch to the next, in order to trace their motions. A major difficulty arises from the fact that a model fit to an individual epoch is not necessarily unique. Choices can be made for the total number of components used, and whether these are delta functions, circular Gaussians, or elliptical Gaussians. We carried out the majority of our fits by initially assuming an elliptical Gaussian for the core component, and circular components for the jet components. This was done to better fit unresolved structure very near the cores, as is sometimes the case with newly emerging components. We found that using circular Gaussians for the jet components reduced the number of free parameters and gave more consistent centroid positions across the epochs.

After each component was fit, we inspected the residual images for regions along the jet above $\sim 5 \mathrm{mJy}$ and iteratively added new components as necessary. If any component (including the core) approached zero axial ratio or an extremely small size during the iterations, we replaced it with a circular Gaussian initially comparable in size to the restoring beam, and then if necessary, a delta-function component. In order to maximize the level of continuity across epochs, we used the model from the previous epoch (with components restored back to initially circular or elliptical Gaussians) as a starting point for the next epoch. Often it was necessary to add or delete components as new jet features emerged and others faded with time below our sensitivity level. We discuss the errors in our fitted components in Section 2.2.3.

\subsubsection{Core Component Identification}

Radio-loud blazars typically have a parsec-scale morphology that consists of a faint jet and a bright, compact core component that is optically thick at centimeter wavelengths. Because the phase self-calibration process removes any absolute positional information from the data, the identification of this component is crucial for kinematic studies, as it provides the (assumed) stationary reference point for all the epochs. In the case of the MOJAVE sample, most jets were highly core dominated, which greatly simplified the identification of the core component. It was typically the brightest feature at the extreme end of a onesided jet at most or all epochs. However, in some of the two-sided jets (discussed in Section 3.6) and in a few individual sources described below, the core component identification was not as straightforward.

$0202+149$. This source contains several jet components within $\sim 0.5$ mas of the bright eastern feature. High-resolution $7 \mathrm{~mm}$ VLBA images by Marscher et al. (2002) indicate a strong component 0.5 mas from the core, which corresponds to the brightest feature in our $2 \mathrm{~cm}$ images. Since there is little evidence of a counterjet in VLBA images published at other wavelengths, and the source is a core-dominated, moderately high-redshift quasar, we assigned the core position to the easternmost feature in the jet.

$0212+735$. This AGN ejected a very bright moving feature in early 1991 that has remained significantly brighter than the westernmost feature. Previous $7 \mathrm{~mm}$ VLBA observations by Lister (2001) confirm the identification of the latter feature as the core.

$0316+413(3 C 84)$. The central feature of this two-sided radio galaxy contains complex, curved structure on scales smaller than 2 mas. There is still uncertainty as to the exact location of the 
core within this structure, even at shorter wavelengths (Dhawan et al. 1998). We have arbitrarily chosen the northernmost feature within the central complex as the core component.

$0333+321$. There is a significant small-scale structure near the westernmost feature in the jet, both at $2 \mathrm{~cm}$ and at longer wavelengths (Lister \& Smith 2000). Since there is no evidence of a counterjet in this source at $2 \mathrm{~cm}$ or other wavelengths, we have assigned the core identification to the westernmost feature, despite the fact that it is not the brightest feature at all epochs.

$0738+313$. Prior to 2001 , the jet was dominated by a bright southern feature, which subsequently faded. We consider the northernmost feature to be the core of this source.

$0742+103$. This is the only bona fide peaked-spectrum source in the flux-density-limited MOJAVE sample (Torniainen et al. 2005), and given the known characteristics of this class, it is a potential candidate for a two-sided jet structure. It is among the highest-redshift objects in the MOJAVE sample $(z=2.624)$. We have fit a slightly weaker component to the southeast of the brightest feature at all epochs, but have arbitrarily assigned the core position to the brightest feature in the images. We were not able to find any published shorter wavelength VLBI images that could be used to clarify the structure near the core.

$0923+392$. This well-studied core-dominated blazar has a bright complex of eastern jet components that have been interpreted as a region where the jet bends directly into the line of sight. VLBI spectral analysis by Alberdi et al. (1997) has identified the (weaker) westernmost jet feature as the core.

$1226+023$ (3C 273). This well-known optically lowpolarization quasar frequently ejects very bright components that dominate the core emission. We have assigned the core position to the northeastern-most jet feature at all epochs.

$1228+126$ (M87). This nearby radio galaxy has a relatively faint diffuse extension to the east of the brightest feature in the jet, which Ly et al. (2007) and Kovalev et al. (2007) argued could be a counterjet. We have assigned the core position to the brightest feature, since it has the highest brightness temperature and is also the site of weak circularly polarized emission (Homan $\&$ Lister 2006). The kinematic fit that we present here differs slightly from that of Kovalev et al. (2007), since we have not used the 2005 April 21 epoch which lacked the St. Croix antenna.

$1548+056$. The north-south jet direction and poorer $(u, v)$ coverage of this equatorial source make its core identification difficult. We have assigned the core to the southernmost jet feature.

1958-179. There are several components within the innermost 1 mas of the jet. Given the difficulty in resolving the emission on this small scale, we have assigned the core position to the brightest feature at all epochs.

$2021+614$. This radio galaxy has long been considered part of the gigahertz-peaked spectrum class; however, owing to its longterm variability at high frequencies and shallow spectral curvature, we have classified it as a flat-spectrum source. Archival multi-frequency VLBA and space-VLBI observations (Lister et al. 2001) suggest the core is located in the southernmost feature, which has a flat spectral index and high brightness temperature.

2128-123. This core-dominated, optically low-polarization quasar is too strongly variable for the peaked spectrum class (Torniainen et al. 2005), despite having a radio spectrum that is slightly peaked (Kovalev et al. 1999). We have assigned the core position to the northeastern-most feature in the jet.

$2134+004$. This source has a significantly curved ridgeline and highly complex polarization structure (Lister \& Homan
2005; Homan \& Lister 2006). Shorter-wavelength VLBA observations by Lister \& Smith (2000) indicate that the jet core lies at the southeastern edge of the radio structure.

$2251+158(3 C 454.3)$. This powerful quasar undergoes frequent flares and component ejections that dominate the core emission. We have assigned the core position to the easternmost jet feature at all epochs.

\subsubsection{Jet Feature Identification Across Epochs}

In most cases, the identification of jet features across epochs proved to be relatively straightforward, owing to the slow angular evolution of the source with time. We also employed additional checks on the continuity of flux density and brightness temperature over time to confirm the cross-identifications. In some cases, however, the situation was much more difficult because of one or more of the following factors: (1) insufficiently frequent temporal coverage, (2) jet features with extremely fast angular speeds and/or flux density decay timescales, (3) a high rate of new jet feature ejection, (4) jets with significant small-scale (possibly curved) structure at or below the VLBA resolution level, and (5) jets containing both moving and nearstationary features. Piner et al. (2007) discussed how temporal under-sampling can often lead to component misidentification and incorrect estimates of apparent jet speeds in AGNs.

In Table 2, we list all of the robust components that we consider to have reliable identifications across a minimum of five epochs. We point out that despite the fact that some features in the list of all fitted components (Table 1) have been assigned the same ID number across epochs, only those listed in Table 2 have robust cross-identifications. Many of these were the result of comparing fits made completely independently by two or more authors, who were free to make their own choices regarding their fits and cross-identifications within the general guidelines described above.

There were eight sources in the sample for which we were not able to measure any robust jet speeds: $0109+224,0235+164$, $0727-115,0742+103,1124-186,1324+224,1739+522$, and 1741-038. Three of these jets were highly compact, with little resolved structure (see Section 3.7). The sources $0235+164$, $1324+224$, and $1741-038$ were all extremely compact, with no measured components at separations larger than 0.4 mas. The components we did observe had considerable scatter in their fitted positions, and none were robust. Given that the structure in these jets was on angular scales just at or less than the size of the restoring beam, we cannot quote any reliable upper limits on their apparent speeds. Their kinematics would be better studied with shorter wavelength ground and/or space VLBI, which could provide better angular resolution. The other five jets for which we were unable to measure robust speeds were:

$0109+224$. This is a BL Lac object with an unknown redshift. A lower limit of $z=0.4$ was claimed by Falomo (1996) on the basis of its optical host galaxy appearance. The fitted jet components display an unusual amount of positional jitter from epoch to epoch, which may be a result of temporally undersampled fast motions.

0727-115. There was too much positional scatter in the components to consider any of them as robust. Piner et al. (2007) analyzed 19 VLBI epochs at $4 \mathrm{~cm}$ and found three components with different speeds, all with very large error values. Kellermann et al. (2004) reported a very high speed of $31.2 \pm 0.6 c$ but this was based on only three epochs at $2 \mathrm{~cm}$. 
Table 2

Jet Kinematics

\begin{tabular}{|c|c|c|c|c|c|c|c|c|c|c|c|c|c|c|c|}
\hline Source & $(2)$ & (3) & $\begin{array}{c}\langle S\rangle \\
(\mathrm{mJy}) \\
(4)\end{array}$ & $\begin{array}{c}\langle R\rangle \\
\text { (mas) } \\
(5)\end{array}$ & $\begin{array}{c}\langle\vartheta\rangle \\
\text { (deg) } \\
(6)\end{array}$ & $\begin{array}{c}\mu \\
\left(\mu \text { as yr }^{-1}\right) \\
(7)\end{array}$ & $\begin{array}{c}\beta_{\text {app }} \\
c \\
(8) \\
\end{array}$ & $\begin{array}{c}\phi \\
\text { (deg) } \\
(9)\end{array}$ & $\begin{array}{c}|\langle\vartheta\rangle-\phi| \\
(\operatorname{deg}) \\
(10) \\
\end{array}$ & $\begin{array}{c}\dot{\mu}_{\|} \\
\left(\mu \text { as yr }^{-2}\right) \\
(11)\end{array}$ & $\begin{array}{c}\dot{\mu}_{\perp} \\
\left(\mu \mathrm{as} \mathrm{yr}^{-2}\right) \\
(12)\end{array}$ & $\begin{array}{r}T_{e j} \\
\text { (13) } \\
\end{array}$ & $\begin{array}{l}T_{\text {mid }} \\
\text { (14) }\end{array}$ & $\begin{array}{c}\Delta \alpha \\
\text { (mas) } \\
(15)\end{array}$ & $\begin{array}{c}\Delta \delta \\
\text { (mas) } \\
\text { (16) } \\
\end{array}$ \\
\hline \multirow[t]{4}{*}{$0003-066$} & 1 & 14 & 176 & 6.7 & 278.9 & $135 \pm 11^{\mathrm{a}}$ & $2.89 \pm 0.24$ & $236.9 \pm 8.3$ & $42.0 \pm 8.3^{b}$ & $-75.6 \pm 11$ & $-13.1 \pm 14$ & & 2004.37 & 0.07 & $\overline{0.12}$ \\
\hline & 3 & 12 & 163 & 1.3 & 255.2 & $101 \pm 9^{\mathrm{a}}$ & $2.17 \pm 0.19$ & $263.9 \pm 11$ & $8.8 \pm 11$ & $29.4 \pm 11$ & $79.1 \pm 15$ & $\ldots$ & 2005.03 & 0.05 & 0.07 \\
\hline & 4 & 11 & 507 & 0.8 & 193.1 & $41 \pm 8$ & $0.88 \pm 0.17$ & $180.8 \pm 9.2$ & $12.3 \pm 9.2$ & $0.5 \pm 10$ & $-14.1 \pm 6.6$ & $1987.3 \pm 7.2$ & 2005.03 & 0.03 & 0.04 \\
\hline & 5 & 7 & 234 & 0.5 & 282.6 & $123 \pm 16$ & $2.64 \pm 0.34$ & $229.6 \pm 8.6$ & $53.0 \pm 8.9^{\mathrm{b}}$ & $\ldots$ & $\ldots$ & & 2005.62 & 0.02 & 0.06 \\
\hline $0007+106$ & 1 & 6 & 336 & 0.4 & 290.0 & $166 \pm 16$ & $0.971 \pm 0.094$ & $291.1 \pm 2.8$ & $1.1 \pm 3.1$ & $\ldots$ & $\ldots$ & $2003.67 \pm 0.24$ & 2005.86 & 0.04 & 0.01 \\
\hline $0016+731$ & 1 & 12 & 144 & 1.1 & 137.3 & $87 \pm 5$ & $6.74 \pm 0.39$ & $152.1 \pm 2.6$ & $14.7 \pm 2.8^{\mathrm{b}}$ & $4.5 \pm 2.9$ & $4.9 \pm 2.3$ & $\ldots$ & 2000.63 & 0.04 & 0.07 \\
\hline $0048-097$ & 1 & 9 & 22 & 1.4 & 357.9 & $344 \pm 71$ & $\ldots$ & $7.8 \pm 4.0$ & $10.0 \pm 4.2$ & $\ldots$ & $\ldots$ & $2003.31 \pm 0.83$ & 2004.53 & 0.08 & 0.27 \\
\hline \multirow[t]{4}{*}{$0059+581$} & 2 & 13 & 21 & 1.5 & 240.8 & $197 \pm 14^{\mathrm{a}}$ & $7.28 \pm 0.52$ & $253.2 \pm 4.1$ & $12.4 \pm 4.3$ & $48.7 \pm 13$ & $-1.6 \pm 14$ & $\ldots$ & 2004.00 & 0.11 & 0.12 \\
\hline & 3 & 10 & 84 & 0.8 & 234.2 & $283 \pm 20$ & $10.46 \pm 0.74$ & $248.7 \pm 3.8$ & $14.5 \pm 4.0^{\mathrm{b}}$ & $-19.6 \pm 45$ & $-13.8 \pm 43$ & $\ldots$ & 2005.95 & 0.07 & 0.06 \\
\hline & 4 & 10 & 155 & 0.6 & 196.2 & $162 \pm 13$ & $5.99 \pm 0.48$ & $230.7 \pm 4.8$ & $34.4 \pm 5.0^{\mathrm{b}}$ & $-35.2 \pm 28$ & $9.9 \pm 30$ & & 2005.95 & 0.04 & 0.05 \\
\hline & 5 & 6 & 290 & 0.4 & 164.7 & $300 \pm 23$ & $11.09 \pm 0.85$ & $166.4 \pm 4.2$ & $1.7 \pm 4.4$ & $\ldots$ & $\ldots$ & $2005.88 \pm 0.17$ & 2007.01 & 0.02 & 0.02 \\
\hline \multirow[t]{3}{*}{$0106+013$} & 1 & 6 & 40 & 3.0 & 237.0 & $311 \pm 46$ & $26.5 \pm 3.9$ & $228.9 \pm 8.4$ & $8.1 \pm 8.5$ & $\ldots$ & $\ldots$ & $1996.2 \pm 2.0$ & 2005.33 & 0.15 & 0.15 \\
\hline & 2 & 6 & 58 & 1.7 & 251.7 & $286 \pm 45$ & $24.4 \pm 3.8$ & $263.1 \pm 2.0$ & $11.4 \pm 2.2^{\mathrm{b}}$ & $\ldots$ & $\ldots$ & $\ldots$ & 2005.33 & 0.15 & 0.03 \\
\hline & 3 & 7 & 562 & 0.8 & 232.6 & $249 \pm 9$ & $21.22 \pm 0.77$ & $239.4 \pm 3.0$ & $6.8 \pm 3.1$ & $\ldots$ & $\ldots$ & $2002.30 \pm 0.14$ & 2005.33 & 0.02 & 0.05 \\
\hline $0119+115$ & 1 & 9 & 72 & 3.1 & 6.7 & $513 \pm 20$ & $17.10 \pm 0.67$ & $1.4 \pm 2.4$ & $5.2 \pm 2.5$ & $\ldots$ & $\ldots$ & $1998.43 \pm 0.48$ & 2005.03 & 0.10 & 0.09 \\
\hline
\end{tabular}

Notes. Columns are as follows: (1) IAU name (B1950.0), (2) component number, (3) number of fitted epochs, (4) mean flux density, (5) mean distance from the core component, (6) mean position angle with respect to the core component, (7) magnitude of fitted angular velocity vector, (8) fitted speed in units of the speed of light, (9) position angle of velocity vector, (10) offset between the mean position angle and velocity vector position angle, (11) acceleration parallel to the velocity direction, (12) acceleration perpendicular to the velocity direction, (13) fitted ejection date, (14) date of reference (middle) epoch used for fit, (15) right ascension error of individual epoch positions (mas), (16) declination error of individual epoch positions (mas).

${ }^{a}$ Component shows significant acceleration.

${ }^{\mathrm{b}}$ Component shows significant non-radial motion

${ }^{\mathrm{c}}$ Component shows significant inward motion.

${ }^{\mathrm{d}}$ Component is unusually slow (LPS; see Section 3.5 ).

(This table is available in its entirety in machine-readable and Virtual Observatory (VO) forms in the online journal. A portion is shown here for guidance regarding its form and content.) 
$0742+103$. This source likely underwent a structural change in the core region in the time period 2002-2004, during a temporal gap in our VLBA observations. We are not able to measure any robust component speeds in the jet because of uncertainties in the core position over time. The parsec-scale jet structure is approximately stable since 2005 , which is consistent with the low speed $(2.7 \pm 0.9 c)$ reported by Kellermann et al. (2004) at $2 \mathrm{~cm}$. The three component speeds reported by Piner et al. (2007) at $4 \mathrm{~cm}$ have large error values.

1124-186. The jet structure in this low-declination source is very compact and extended in a southern direction that unfortunately coincides with the major axis of the restoring beam. The fitted component positions thus suffer from high scatter, and we categorized none of them as robust. Piner et al. (2007) reported two component speeds of $1.8 \pm 29.5 c$ and $4.0 \pm 5.4 c$ for this jet at $4 \mathrm{~cm}$.

$1739+522$. The jet is highly curved near the core, and contains considerable small-scale structure that made it difficult to crossidentify its components across the epochs at the level of our angular resolution. The single component reported by Piner et al. (2007) at $4 \mathrm{~cm}$ had an inward speed of $16.8 \pm 7.9 c$.

\subsubsection{Errors on Fit Parameters}

The errors in the model fit parameters of individual Gaussian components in VLBA images are difficult to determine, because of nonlinear dependencies on the interferometric array coverage, thermal noise, and the presence of other nearby jet features. For isolated components, our previous work (see Appendix A of Homan et al. 2002) has indicated typical errors of 5\% on the total intensities and positional errors of $\sim 1 / 5$ of the restoring beam dimension. For the brightest components, these errors are smaller by approximately a factor of 2 .

The vector motion (i.e., constant angular velocity) and acceleration models described in Section 3.2 were fit to our position versus time data using a $\chi^{2}$ minimization routine (Press et al. 2007). Given the well understood difficulties in obtaining robust and statistically accurate component position uncertainties directly from the interferometric data in each epoch independently, we followed the approach used by Homan et al. (2001) of initially assuming equal weighting for all of our data points in a given fit, and then uniformly rescaling their weights such that $\chi^{2}=N_{\text {points }}-N_{\text {parameters }}$. In addition to the limitation that all data points are equally weighted, this approach has the disadvantage that the final, minimum $\chi^{2}$ value itself is fixed to equal the number of degrees of freedom, and therefore it cannot be used to determine the suitability of the chosen motion model. However, under the assumption that the applied model is appropriate, this approach does yield good uncertainty estimates in all of the model parameters.

We have studied the scatter of the measured component positions about the best-fit proper motion model: this was either the initial vector motion fit, or the acceleration model if the measured acceleration was $\geqslant 3 \sigma$. Estimates of the typical component position uncertainties appear in the last two columns of Table 2. In Figure 1, we plot the distribution of these $x$ and $y$ component position uncertainties. The individual distributions for $x$ and $y$ are so similar that we have simply combined the data to construct Figure 1. We find the most probable component positional uncertainty is in the range 0.04-0.06 mas, with 68\% of the components having an estimated positional uncertainty $\leqslant 0.12$ mas and $95 \% \leqslant 0.29$ mas.

An alternate means of estimating the component positional errors (e.g., Piner et al. 2007) is to examine the differences in

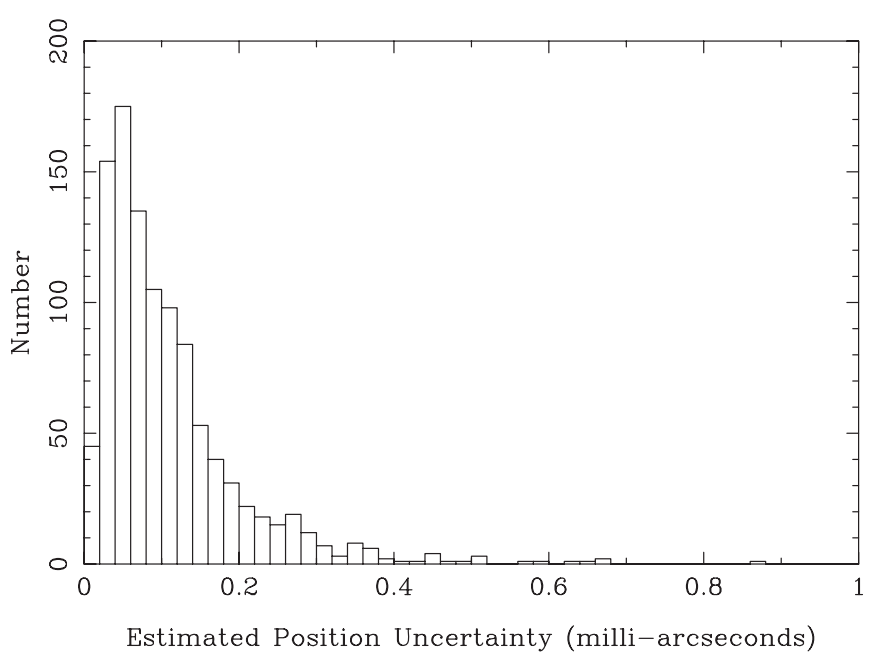

Figure 1. Histogram of the estimated typical position uncertainty for fitted Gaussian jet components. A total of 1052 component uncertainties are plotted ( 2 for each of the 526 robust components in our sample, one in the $x$-direction and the other in the $y$-direction). The large $x$-position uncertainties for components 1 and 2 of $1228+126$ (M87; 1.01 and 1.32 mas, respectively) are not plotted.

the fits to two or more individual epochs that are very closely spaced in time, such that the jet has approximately the same structure in both data sets. We have done this analysis on 10 pairs and one triplet of epochs on several MOJAVE sources that were within seven days of each other, and were of similar data quality. Based on the fastest observed proper motion in each source, the expected real component movement between the epochs in each of the pairs is less than 0.01 mas for all the sources except for BL Lac, which has an estimated movement of 0.04 mas. Therefore, the differences in the fitted component positions between the epochs should effectively reflect the positional errors of the components.

The positional difference distribution of the individual fitted components has a narrow Gaussian central peak at zero and non-Gaussian tails up to roughly 0.6 mas. In terms of their $x$ (R.A.) coordinates, approximately $68 \%$ of the components have a positional uncertainty smaller than 0.08 mas, or roughly $10 \%$ of the beam size. In the $y$ (declination) direction, approximately $95 \%$ of the components have a positional uncertainty smaller than 0.33 mas (roughly $30 \%$ of the beam size).

We find that the positional accuracy depends on the size of the component and, to some degree, on its flux density (i.e., signal-to-noise ratio $(\mathrm{S} / \mathrm{N}))$. The positional difference distribution, measured in units of component size convolved with the beam size, is very close to Gaussian. For extended components, a $68 \%$ (95\%) confidence limit is $7 \%(22 \%)$ of the component size convolved with the beam size. For a pointsource or delta-function component with good $\mathrm{S} / \mathrm{N}$, the $1 \sigma$ uncertainty in position is less than one tenth the beam size. These error estimates are consistent with those derived from our multi-epoch fits as described above.

\section{PARSEC-SCALE JET KINEMATICS}

\subsection{Vector Speeds}

For our initial velocity analysis of the robust features, we have assumed non-accelerating, two-dimensional motions on the sky. We fit vector proper motions to the $x$ and $y$ positions of all 526 robust components in our sample, initially assuming simple, non-accelerating motion. For those features with at least 
10 epochs of data, we also refit the data including acceleration terms, as described in Section 3.2, and in more detail by Homan et al. (2009). The resulting fits are velocity vectors relative to the component's position at a reference (middle) epoch listed in Column 14 of Table 2. This table lists the magnitude, $\mu$, of this vector (Column 7) in microarcseconds per year, and its direction on the sky, ( $\phi$; Column 9$)$, as well as the leastsquare fit errors on these quantities. Note that for components with $\geqslant 3 \sigma$ accelerations, the values for $\mu$ and $\phi$ in Table 2 are from the fit with the acceleration terms included; however, as described in Section 3.2, these correspond closely to the same values obtained from the unaccelerated fit.

In order to characterize whether the motion is directed purely radially toward or away from the core, we compare $\phi$ with the mean position angle of the component ( $\langle\vartheta\rangle$; Column 6$)$. We consider features in which the velocity is significantly offset from the purely radial (i.e., $|\langle\vartheta\rangle-\phi|=0^{\circ}$, or $|\langle\vartheta\rangle-\phi|=180^{\circ}$ ) direction at the $3 \sigma$ level to be "non-radial." These are flagged in Column 10. We flag features as "inward" in Column 9 if the velocity vector offset is significantly in excess of $90^{\circ}$. We discuss these rare inwardly moving features in more detail in Section 3.4.

In Column 13, we list the time of ejection (defined as when the core separation equals zero) derived from the vector fit. No times of ejection are listed for features in which any of the following apply: (1) the component shows significant acceleration (see Section 3.2), (2) the magnitude of the vector motion is smaller than three times its associated error, or (3) the motion is significantly non-radial or inward.

\subsection{Accelerations}

For the features in which we have obtained at least 10 epochs, there is sufficient positional information to attempt a simple twodimensional acceleration fit. The method is described by Homan et al. (2001), and uses the following parameterization:

$$
\begin{aligned}
& x(t)=\mu_{x}\left(t-t_{x 0}\right)+\frac{\dot{\mu}_{x}}{2}\left(t-t_{\text {mid }}\right)^{2} \\
& y(t)=\mu_{y}\left(t-t_{y 0}\right)+\frac{\dot{\mu}_{y}}{2}\left(t-t_{\text {mid }}\right)^{2},
\end{aligned}
$$

where $t_{\text {mid }}=\left(t_{\min }+t_{\max }\right) / 2$ is the middle epoch of our observations. This epoch is chosen as the relative epoch for fitting accelerations, and this choice has the benefit that the other parameters of the fit will correspond closely to those from the vector motion fit (which assumes no acceleration). Thus, the mean angular velocities in $x$ and $y$ are given directly as $\dot{x}\left(t_{\text {mid }}\right)=\mu_{x}$ and $\dot{y}\left(t_{\text {mid }}\right)=\mu_{y}$, respectively. The epochs of origin, assuming these average speeds have applied since the component emerged are also given directly by this fit: $t_{x 0}=t_{\mathrm{mid}}-x\left(t_{\mathrm{mid}}\right) / \dot{x}\left(t_{\mathrm{mid}}\right)$ and $t_{y 0}=t_{\mathrm{mid}}-y\left(t_{\mathrm{mid}}\right) / \dot{y}\left(t_{\mathrm{mid}}\right)$.

The angular accelerations on the sky plane are given by $\ddot{x}=\dot{\mu}_{x}$ and $\ddot{y}=\dot{\mu}_{y}$. The accelerations are then resolved into two components relative to the mean angular velocity direction $\phi$ : one parallel component, $\dot{\mu}_{\|}$, and one perpendicular component, $\dot{\mu}_{\perp}$, along $\phi+90^{\circ}$. In this way, $\dot{\mu}_{\|}$directly quantifies changes in apparent speed of the component during our observations, and $\dot{\mu}_{\perp}$ quantifies changes in an apparent direction on the plane of the sky. In a few isolated cases where the angular speed of the feature is extremely slow (less than $\sim 50 \mu \mathrm{as} \mathrm{y}^{-1}$, e.g., component 4 of 1253-055 (3C 279), there may not be a welldefined velocity vector direction. In such cases, the individual perpendicular and parallel components of the acceleration are not necessarily meaningful.
We note that our approach to fitting for angular accelerations assumes that the same acceleration has applied throughout our observing period. Other models, such as impulsive accelerations inducing sudden changes in speed and/or direction are certainly possible and may provide a better fit in some cases, such as component 1 in 1253-055 (3C 279) which was reported to make such a sudden change in speed and direction by Homan et al. (2003).

We list the parallel and perpendicular components of the fitted accelerations in Columns 11 and 12, respectively of Table 2. We have flagged in Column 7 any features which have a parallel or perpendicular acceleration exceeding the $3 \sigma$ level. Of the 311 features we examined, $109(35 \%)$ fall into this category. The angular speed $(\mu)$ listed in Table 2 for these features reflects the value obtained from the acceleration fit, as opposed to the vector one. It is important to note that there are likely other jet features in Table 2 that are in fact changing speed and/or direction, but we cannot discern them as such because of insufficient temporal coverage.

In Figure 2 we plot our fits to each source in a core separation distance versus time diagram. Each robust jet component is indicated by the colored points, which are joined by either a solid line (non-accelerating fit) or a dotted line (accelerating fit). Thin line widths are used in cases where the component motion is significantly non-radial. Unfilled colored circles indicate individual data points that were not used in the kinematic fits, and unfilled black circles indicate non-robust components.

We plot the motion fits and sky positions of the individual robust components for the selected AGNs in Figures 3 and 4. Extended versions of Figures 2-4, containing plots of all of the robust jet components in the MOJAVE sample, are available online. The left-hand panels of Figures 3 and 4 show a $2 \mathrm{~cm}$ VLBA contour image of the source from Paper $\mathrm{V}$ at the middle epoch listed in Table 2. The orange box delimits the zoomed region displayed in the right-hand panels. The component's position at the middle epoch is indicated by the orange crosshairs. The dotted line connects the component's middle epoch position with that of the core feature. The positions at other epochs are plotted with unfilled blue circles. The red solid line indicates the vector (or accelerating) fit (see Table 2) to the component positions. The red arrows in the right-hand panels indicate the direction of motion, and the gray dashed circles/ellipses represent the FWHM sizes of the individual fitted Gaussian components.

\subsection{Non-radial Motions}

In our previous study of AGN jet kinematics in the $2 \mathrm{~cm}$ survey (Kellermann et al. 2004), we identified many cases where jet features were moving in directions that did not extrapolate back to their presumed origin at the base of the jet. These non-radial components provided convincing evidence that jet features change direction, and ruled out simple ballistic "cannon ball" models. In our study of the MOJAVE sample, we find that 166 out of the 526 robust components $(32 \%)$ satisfy our definition of non-radial motion (Section 3.1). A comparable fraction was found in smaller samples by Kellermann et al. (2004) and Piner et al. (2007), indicating that non-radial motion is a common feature of the jet flow in blazars. We note that three of these non-radial components might be considered "inward," i.e., their direction of motion is greater than $90^{\circ}$ from the local jet direction at the $3 \sigma$ level. Five additional components that would qualify as inward do not qualify as non-radial since their 


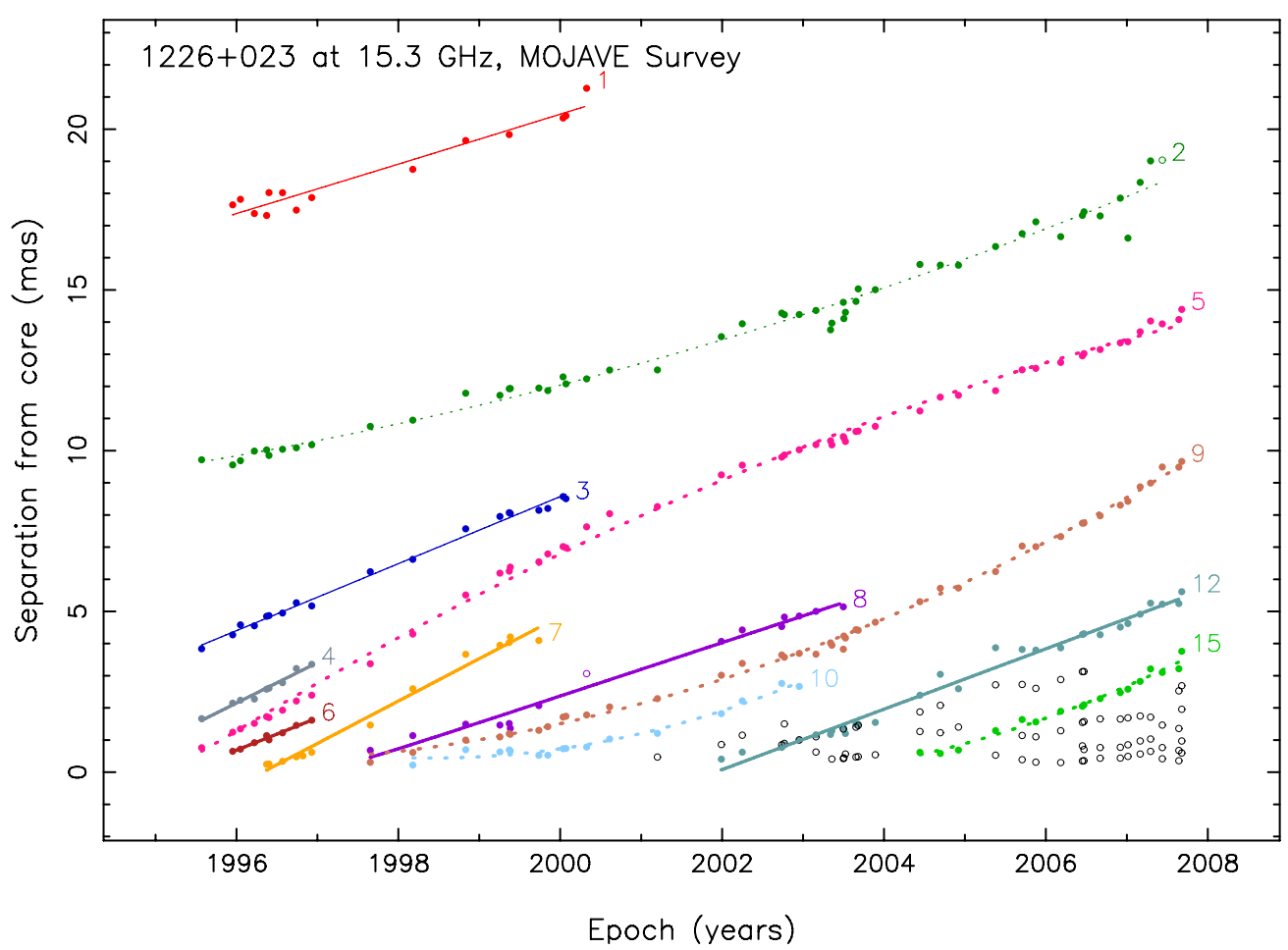

Figure 2. Plot of angular separation from core vs. epoch for Gaussian jet components. The B1950 source name is given at the top left of each panel. Color symbols indicate robust components for which kinematic fits were obtained (dotted and solid lines). The solid lines indicate vector motion fits to the data points assuming no acceleration, while the dotted lines indicate accelerated motion fits. Thick lines are used for components whose fitted motion is along a radial direction from the core, while the thin lines indicate non-radial motions. Unfilled colored circles indicate individual data points that were not used in the kinematic fits, and unfilled black circles indicate non-robust components. The component identification number is indicated next to the last epoch of each robust component.

(The complete figure set (136 images) is available in the online journal.)

$|\langle\vartheta\rangle-\phi|$ values are less than $3 \sigma$ from $180^{\circ}$. We discuss these features in more detail in Section 3.4.

The high incidence of non-radial motion among the components in our sample indicates that changes in jet motion are common. A more detailed analysis of a subset of these data, to be presented by Homan et al. (2009), indicates that in the vast majority of cases, the non-radial motions are indeed caused by accelerations in a direction perpendicular to the velocity vector.

\subsection{Apparent Inward Motions}

For the great majority of our reported jet features, we find that their motion is either directed outward from the core, or is nearly stationary. Fewer than 10 of the 526 robust features we have studied show evidence for inward motion, i.e., where the projected separation between the jet feature and the core decreases with time, and most of these are only marginally significant. The illusion of apparent inward motion can be explained in terms of one or more of the following scenarios:

1. If a newly emerging jet feature and the core are not resolved by our beam, this would cause a temporary shift in the measured position of the core centroid and a corresponding apparent decrease with time in the apparent separation of the core and other more distant jet features (e.g., Gómez et al. 1995).

2. It is also possible that the true core is not seen, possibly due to absorption, and that the only observed features are parts of a jet which are moving with different velocities (e.g., Lobanov 1998). If the furthest component is moving with a slower velocity than the one closest to the obscured core, then the two components will appear to be approaching each other.
3. An apparent decrease in component separation from the core could also arise due to component motion away from the core but along a highly curved jet. If the jet bends back and across the line of sight, the projected separation from the core can appear to be decreasing, even though the actual distance from the core is increasing with time (e.g., Marscher et al. 1991).

4. Some observed jet features may merely reflect patterns in the flow, of which some might even be moving backward. Istomin \& Pariev (1996) have discussed an electronpositron jet model where an observer located close to the direction of motion will see backward-moving knots. Gong (2008) has proposed a model of a precessing jet which illuminates an ambient screen, so that an observer oriented near the plane of the screen can see projected motion in any direction even though the jet itself is always flowing outward. We note, however, that any model involving patterns must account for the very large numbers of observed outward motions, as opposed to inward ones.

5. Internal changes in the brightness distribution of a large, diffuse feature may cause the centroid of the fitted Gaussian to wander, possibly mimicking inward motion.

We discuss several individual cases for inward motion in our sample below.

$1458+718$. This compact steep-spectrum quasar has a very long jet with a bright complex region located about 25 mas from the nucleus as well as a fainter region about 40 mas downstream. Close to the nucleus, as well as for the more distant feature, the motion is in the outward direction with $\beta_{\text {app }} \sim 7$. It is more difficult to accurately determine the motion of the more diffuse 

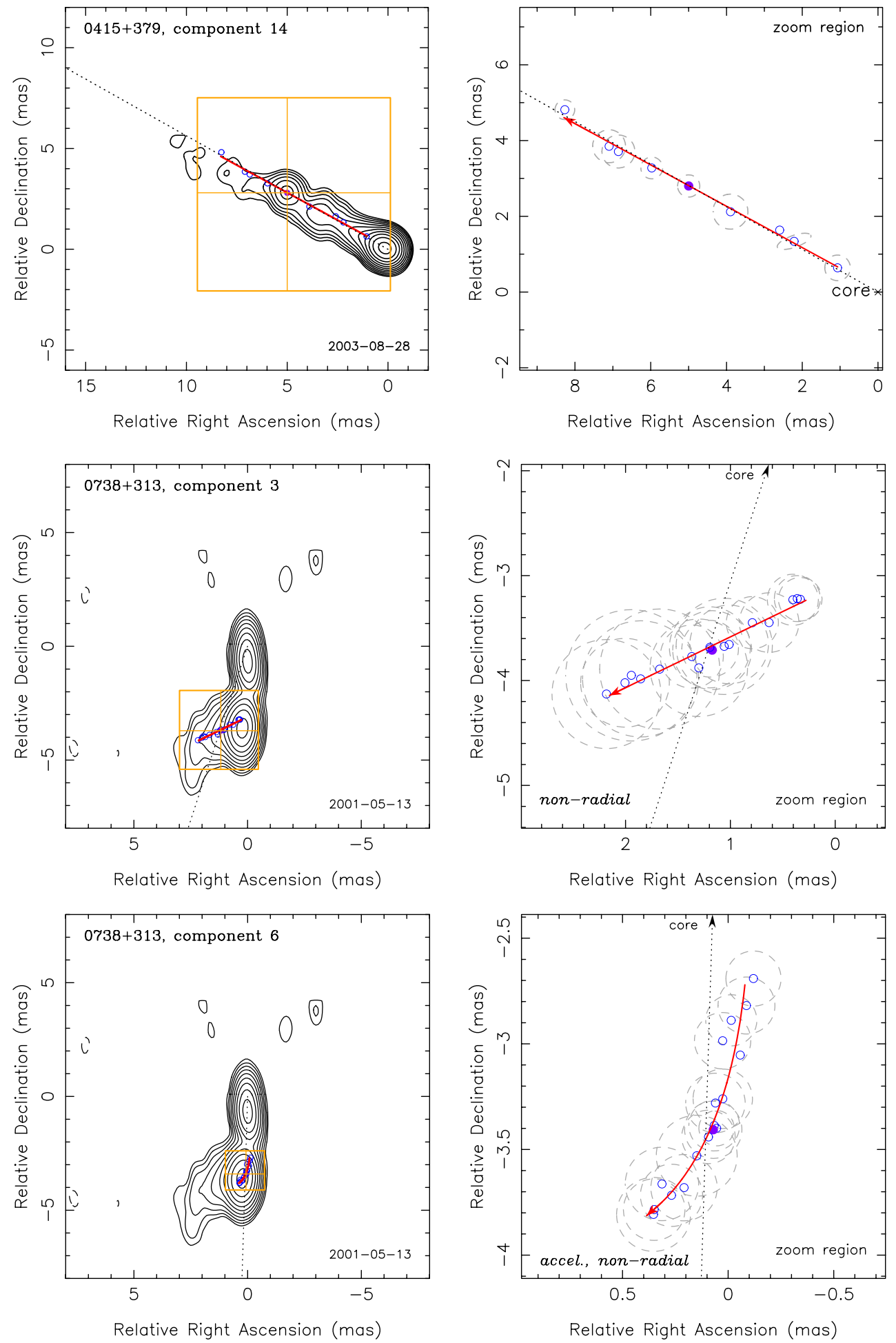

Figure 3. Vector motion fits and sky position plots of individual robust jet features in MOJAVE AGNs. Positions are relative to the core position. The left-hand panels show a $2 \mathrm{~cm}$ VLBA contour image of the source from Paper V at the middle epoch listed in Table 2. The orange box delimits the zoomed region displayed in the right-hand panels. The component's position at the middle epoch is indicated by the orange cross-hairs. The dotted line connects the component with the core feature and is plotted with the mean position angle $\langle\vartheta\rangle$ (Table 2). The position at the middle epoch is shown by a filled violet circle while other epochs are plotted with unfilled blue circles. The red solid line indicates the vector (or accelerating) fit (see Table 2) to the component positions. The red arrows in the right-hand panels indicate the direction of motion, and the gray dashed circles/ellipses represent the FWHM sizes of the individual fitted Gaussian components. Displayed from top to bottom in the figure are component ID = 14 in $0415+379$ ( $3 \mathrm{C} 111), \mathrm{ID}=3$ in $0738+313$, and $\mathrm{ID}=6$ in $0738+313$.

(The complete figure set (526 images) is available in the online journal.)

and complex feature located about 25 mas downstream, but there may be a slight gross inward motion with $\beta_{\text {app }} \sim 2$.

$1803+784$. This is a classical core-jet source. The most distant, somewhat extended, feature is moving away from the core with a velocity of $9.0 \pm 2.5 c$, and two intermediate features appear almost stationary over the past 10 years or more. The two innermost features each appeared to be moving away from the core with a velocity of $\sim 1.5 c$ until the year 2000 , when 

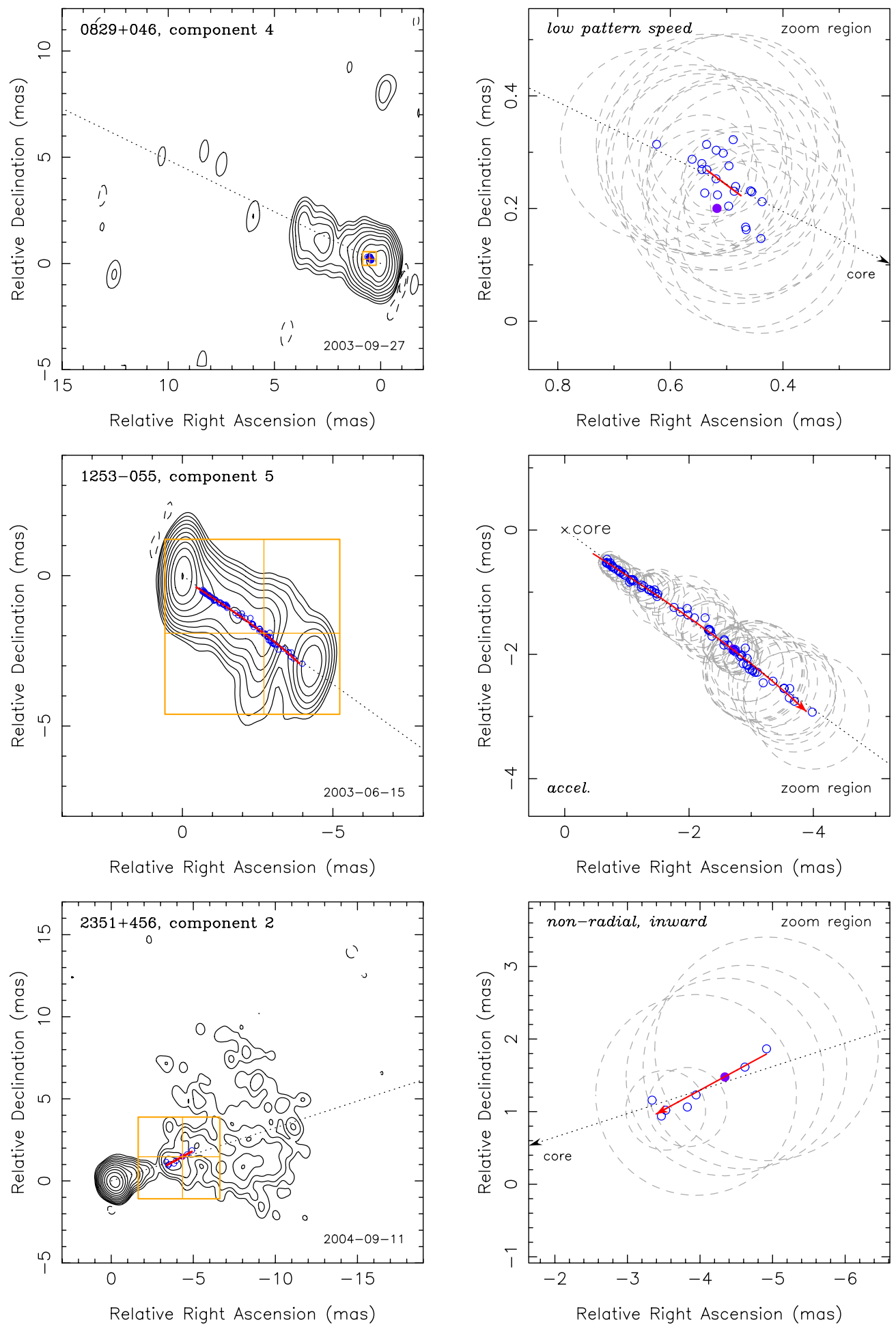

Figure 4. Same as Figure 3. Displayed from top to bottom in the figure are component ID $=4$ in $0829+046$, ID $=5$ in $1253-055$ (3C 279$)$, and ID $=2$ in $2351+456$. (The complete figure set (526 images) is available in the online journal.)

both appeared to slow down. Since 2004, the distance between of each of them and the core has decreased. The very similar apparent motion of these two inner features is characteristic of an apparent change in separation due to an emerging, yet unresolved new component. However, no such feature is evident in VLBA data obtained as of 2009 March.

$2005+403$. There is a pronounced bend in the jet located about 2 mas from the nucleus. Close to the nucleus, there is a bright feature which has moved 0.1 mas closer to the nucleus over the three-year period that it has been observed, but this result depends primarily on the first and last observations in 2004 and 2007, respectively. Between mid 2005 and mid 2006 there was little or no motion. If real, the apparent inward motion of this feature is most likely due to a new emerging component which is unresolved from the core. Beyond about 1 mas, the flow is in the general direction of the jet ridge line. 
$2021+614$. This jet has a slow-moving feature (ID = 2) located approximately 3 mas downstream from the core that is classified as inward according to our formal definition. The fitted angular speed is extremely slow $\left(11 \pm 3 \mu\right.$ as $\left.\mathrm{y}^{-1}\right)$, and although there is a general downward trend in its separation versus time plot, the component oscillates about its mean position over time.

$2200+420$. The innermost component (ID =7) of this source changes position angle from $-160^{\circ}$ to $\sim-170^{\circ}$ from 1999 to 2006, while maintaining a relatively stable separation of 0.3 mas. Since this is at the extreme limit of our resolution, and the component has a low speed (Section 3.5), the vector motion direction is not necessarily well defined. We thus cannot conclude for certain if the motion is truly inward.

$2201+171$. Close to the core, the flow is along the direction of the jet, but 1.4 arcsec downstream, a bright feature appears to flow in the reverse direction, possibly because of confusion with a newly emerging component.

2230+114 (CTA 102). This AGN has an elongated jet with several pronounced twists and bends. Along most of the jet, the flow appears to be close to the jet direction, with apparent velocities between $c$ and $16 c$. A single component at $\sim 6$ mas from the core is moving radially inward at $0.97 \pm 0.22 c$. The contour maps indicate an apparently sharp bend of approximately $50^{\circ}$ in the jet at this location.

$2351+456$. As shown in Figure set 3, 2351+456 has a welldefined jet close to the core which has a sharp nearly rightangle bend about 7 mas downstream, beyond which it opens into a very diffuse structure characteristic of a direct head-on jet. Close to the core and near the bend the flow follows the ridge line with an apparent $\beta_{\text {app }}$ of 10-15. However, a bright feature located about 4 mas downstream is moving toward the core with $\beta_{\text {app }}=28.3 \pm 1.3$. Over the six-year period that we have observed this feature it has moved a total of about 2 mas, so the apparent motion cannot be explained by the emergence of a new feature unresolved from the core. Rather, we suggest that the jet of $2351+456$ is viewed nearly end-on, and that the region near 4 mas is where the jet bends back across the line of sight. VLBA data from the MOJAVE program obtained after 2007 indicate that the component at 4 mas has stopped moving inward, supporting this interpretation.

\subsection{Dispersion of Apparent Speeds Within Individual Jets}

Past multi-epoch VLBI studies of individual radio-loud AGNs have identified instances where the jet displays features that move with a range of apparent speeds (e.g., Piner et al. 2007; Jorstad et al. 2005; Kellermann et al. 2004). Such instances may suggest that the motions of some jet features are not related to the flow, or that the jet is experiencing a change in geometry over time due to precession. Some jet features have also been seen to either remain virtually stationary over time, or move at significantly slower apparent speeds than other features in the same jet (Kellermann et al. 2004; Britzen et al. 2005; Jorstad et al. 2005). These low-pattern speed (LPS) features have sometimes been explained as discrete locations in a bent jet that are at an extremely low viewing angle because of the specific geometry involved (Alberdi et al. 2000). In the specific case of a flow moving relativistically along a helical path, the changing viewing angle is predicted to give rise to Dopplerboosted regions that appear nearly stationary in VLBI images. Alternatively, it is possible that some LPS features represent standing shocks in the flow. These can take the form of recollimation shocks in an initially over-pressurized outflow, and have been successfully reproduced in numerical simulations of

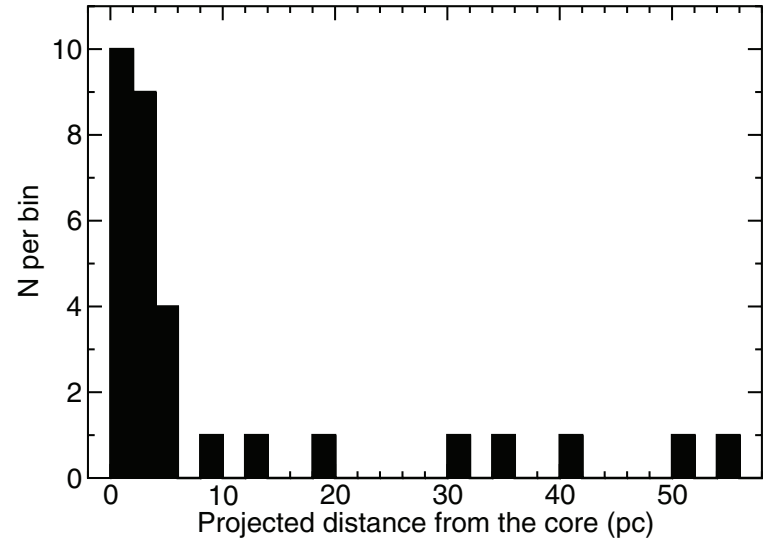

Figure 5. Distribution of projected distance from the core for LPS jet features in the MOJAVE sample.

AGN jets (e.g., Gómez et al. 1995; Perucho \& Martí 2007). Some studies (e.g., Mandal \& Chakrabarti 2008; Becker et al. 2008) have suggested that standing shocks within the unresolved cores of AGN jets may play a major role in accelerating particles near the base of the jet, and could be responsible for the persistent high levels of polarization in blazars (D'Arcangelo et al. 2007; Marscher et al. 2008).

We have examined the distribution of apparent speeds within each jet in the MOJAVE sample, and find a median rms dispersion of $\mathrm{RMS}_{\text {jet }}=0.065$ mas $^{-1}$, or $2.6 c$. We also calculated the median speed value of each MOJAVE jet, and find that the distribution of this value for the entire sample has an rms dispersion of $\mathrm{RMS}_{\text {sample }}=0.29$ mas $^{-1}$, or 7.3c. The $\mathrm{RMS}_{\text {sample }}$ and $\mathrm{RMS}_{\text {jet }}$ values differ by more than a factor of 3 . This supports the previous finding of Kellermann et al. (2004) that most jets generally eject features with a characteristic speed. We found even larger differences between these two RMS values in the cases where (1) the LPS features were dropped from the analysis, or (2) only quasars were considered, or (3) the maximum jet speeds were used to calculate $\mathrm{RMS}_{\text {sample }}$.

We have also used the regular time coverage and completeness of the MOJAVE sample to investigate how prevalent LPS features are in blazars, and whether they are more prone to occur in certain types of AGN jets. We examined all 94 robust features in our sample with radial speeds less than $50 \mu$ as $\mathrm{y}^{-1}$ to see if their motions were non-accelerating and significantly slower than other features in the same jet. We were able to identify only 31 such instances, which are indicated with a flag in Column 5 of Table 2. These LPS features have a bimodal distribution, with the majority lying within projected distances of 0.8 mas $(\sim 6 \mathrm{pc})$ from the core, while only a handful are found further downstream (Figure 5).

Since the close-in LPS features are located approximately within one beamwidth of the core, we have investigated the possibility that they may be artifacts arising from the modelfitting process. We model fitted the sources using different models for the core, including circular and elliptical Gaussians, as well as delta functions, and were not able to eliminate the close-in features. They generally are present in all the epochs on a particular source, except on occasions when they are blended with new components that have emerged from the core. After such components move downstream, the stationary feature then reappears, indicating that it is a persistent feature of the flow. We have also compared our model fits to five jets in common with the Jorstad et al. (2005) sample, who model fitted several closely spaced VLBA epochs at $7 \mathrm{~mm}$ with three times better angular 
resolution. In each case, they identified a stationary component at roughly the same core distance as in our fits (taking into account a typical core shift between these wavelengths of $\sim 0.1$ mas; Kovalev et al. 2008). Jorstad et al. (2005) found in some cases additional stationary features even closer to the core ( $r \simeq 0.1$ mas), which are below the resolution level of our $2 \mathrm{~cm}$ images. Despite our limited sensitivity to these extreme close-in features, we conclude that LPS features are very rarely found in blazar jets at projected distances greater than $6 \mathrm{pc}$ downstream of the core. Given the small viewing angles of the MOJAVE jets, this translates into an approximate de-projected distance of $50 \mathrm{pc}$.

Given the caveats of our limited angular resolution, we find that the incidence rate of LPS features is much higher for the BL Lacs in the sample (10 of 22), as compared to the quasars (15 of 86). Although the latter generally have higher redshifts than the BL Lacs, we find that for the whole MOJAVE sample, $\mathrm{K}-\mathrm{S}$ tests indicate no significant differences in the redshift or $2 \mathrm{~cm}$ VLBA luminosity distributions of the AGN jets with LPS features and those without. It is therefore possible that the conditions in BL Lac jets are more conducive to the formation of strong, stable re-collimation shocks. Numerical hydrodynamical jet simulations by Gómez et al. $(1995,1997)$ have shown that the strength and separation of jet knots associated with recollimation shocks are dependent on the jet opening angle and Mach number, as well as the external density gradient.

\subsection{Two-sided Jets}

Although it is thought that quasars and radio galaxies have intrinsically two-sided jets which feed the distant radio lobes, the great majority of the sources in the MOJAVE sample have one-sided jets, which is not unexpected considering the expected large differential Doppler boosting of the approaching and receding jets. A few sources, however, show two-sided jets; these are mostly low-luminosity radio galaxies, and it is tempting to consider that these sources lie close to the plane of the sky so that there is little differential Doppler boosting. However, Cohen et al. (2007) have shown that the observed number of slowly moving low-luminosity two-sided jets is considerably greater than that would be expected if the distribution were determined by orientation alone, so that the two-sided jets may reflect a population intrinsically different from the one-sided population.

One challenge in determining independent speeds in a twosided jet is determining the unambiguous location of the common, presumably stationary, central core presumed to lie at the base of each jet. We have been able to obtain robust speed measurements of both jet and counterjet components for five twosided jets in our sample. Stacked images over all epochs for these two-sided sources are shown in Paper V and are discussed below.

0238-084 (NGC 1052). This is a bright nearby elliptical galaxy that harbors a well-studied low-luminosity AGN. Vermeulen et al. (2003); Kellermann et al. (2004); Kadler et al. (2004) have previously discussed the observed outflow in two nearly symmetric jets containing multiple features which propagate away from a central region with relatively slow subluminal velocities. Very Large Array (VLA) observations show the jets extending symmetrically out to $1.5 \mathrm{kpc}$ on either side (Cooper et al. 2007; Wrobel 1984). The central region is surrounded by a rich molecular $\left(\mathrm{OH}\right.$ and $\left.\mathrm{H}_{2} \mathrm{O}\right)$, atomic, and ionized gas cloud. $\mathrm{H}$ I observations indicate the presence of three velocity streams, and multi-frequency VLBA observations show patchy free-free absorption, which may come from the torus surrounding the central black hole (Vermeulen et al. 2003; Kadler et al. 2004). The analysis of the jet motions is complicated by two factors. First, the presumed base of the jets is obscured by free-free absorption, especially on the receding side, and second, the simultaneous presence of many features in each jet exacerbates the problem of cross-identifying components over different epochs. We have estimated the location of a virtual center by assuming it is located half way between pairs of symmetrically moving components in opposing jets. Figure set 3 shows the location of each feature that we were able to identify as a function of time. Over the past decade, a new feature is ejected at an average rate of about once per year, each moving with an apparent velocity in the range of $0.1-0.25 c$.

$0316+413(3 C 84)$. NGC 1275 is a prominent radio galaxy that contains a bright complex central core, a southern cocoonlike expanding cloud which is connected to a central feature by a thin faint jet, as well as an inverted spectrum counter feature (Dhawan et al. 1998; Walker et al. 2000). It was detected in $\gamma$-rays during the first four months of the Fermi observatory, during which time $2 \mathrm{~cm}$ MOJAVE VLBA observations indicated a significant brightening of the core region (Abdo et al. 2009). We have determined the motion in the southern lobe to be $\mu=0.27 \pm 0.05$ mas $^{-1}(v=0.31 \pm 0.06 c)$ while the northern lobe appears to move more slowly with $\mu=0.07 \pm 0.02$ mas $^{-1}(0.08 \pm 0.03 c)$. Assuming the jets are intrinsically symmetric, these observed speeds correspond to a Lorentz factor of 0.6 with the motion oriented at about $11^{\circ}$ to the line of sight. However, the central component itself has a complex morphology, with observed motions up to $0.2 c$ or more (Dhawan et al. 1998), which introduces some additional uncertainty in the derived motions of the two outer features. We have taken the origin of motion to be at the northern end of the central feature, as suggested by the $7 \mathrm{~mm}$ VLBA images and corresponding motions within the central feature (Dhawan et al. 1998).

$1228+126$ (Virgo A). M87 harbors a prominent jet which has been imaged at radio, optical, and X-ray wavelengths. We have taken the core to be coincident with the brightest radio component, which is supported by the circular polarization reported at this position (Homan \& Lister 2006). We are able to identify six features along the jet and one counterjet feature which have existed continuously over the decade-long interval covered by our observations. As we reported previously (Kovalev et al. 2007), we find no evidence for any motion faster than a few percent of light speed, with the fastest feature measured at $0.05 c \pm 0.02 c$. The counterjet may be moving at about $0.01 c$, but this is not well determined because of its complex structure. Faster angular motions corresponding to $\sim 2 c$ have been reported by Acciari et al. (2009) in closely temporally spaced $7 \mathrm{~mm}$ VLBA observations, but these are still not sufficient to completely account for the large jetto-counterjet ratio in regions 3 mas downstream of the core by Doppler boosting alone. Therefore, the bulk plasma flow speed must be much greater than the observed pattern motions (Kovalev et al. 2007), and there is the additional possibility that the M87 jet is intrinsically asymmetric. We note that the extreme proximity and low luminosity of M87 set it apart from the other AGN in our sample, which are typically powerful FR-II class radio sources.

$1413+135$. This is a complex, highly unusual two-sided BL Lac object located within an apparent spiral host galaxy (Véron-Cetty \& Véron 2006). Like NGC 1052, the AGN is surrounded by a cloud of molecular, atomic, and ionized gas (Perlman et al. 2002), but $1413+135$ is a thousand times more 
luminous than the AGN in NGC 1052. We have identified seven robust jet features in $1413+135$, two of which are located toward the northeast in an apparent counterjet. Both counterjet features are weak, and so it is difficult to determine their motions with high precision. Based on 10 measurements over a sevenyear period, we find a best-fit velocity of the more distant counterjet feature (ID $=1$ ) of $1.34 \pm 0.56 c$, but we cannot exclude the possibility that this feature is stationary. The other counterjet feature (ID $=2)$ is slow $(0.14 \pm 0.28)$ and is also possibly stationary. By comparison, in the main jet, there are several bright, robust features with apparent speeds ranging from near stationary for the strongest feature to $1.8 \pm 0.2 c$ for the westernmost component (ID $=3$ ).

$1957+405$ (Cygnus A). Cygnus A is a classical (archetypical) FR II radio galaxy. The central compact radio source lies at the origin of the well-known prominent kiloparsec jet structure. It has a luminosity comparable to other powerful AGNs, but like the M87 AGN, the Cygnus A AGN is many orders of magnitude less luminous than the extended jets and lobes. Also, as in M87, the jets are asymmetric, with the western jet being brighter on scales of few parsecs to a hundred kiloparsecs. Interestingly, the ejection dates of the counterjet components match up well with their counterparts in the main jet, indicating near-simultaneous ejections. On the bright, presumably approaching side, the jet appears to be moving with a velocity close to $0.2 c$ and on the faint receding side, $0.1 c$. Assuming that the two sides are intrinsically similar, the intrinsic velocity is about $20 \%$ of the speed of light, with the jets oriented at an angle near $70^{\circ}$ to the line of sight. Multi-wavelength VLBA studies by Bach et al. (2005) report evidence for an absorbing feature in front of the receding jet.

All of the apparent two-sided jets in our flux-density-limited sample are found in relatively low-luminosity and relatively nearby radio galaxies. We have not found any symmetric parsecscale jets in any quasars, although 101 out of the 135 sources in our sample are classified as quasars. These two-sided jets provide additional evidence that the apparent motions do indeed reflect the underlying jet flow and are not simply a (shock) pattern motion which propagates within a relatively stable or slowly moving plasma. In the case of random pattern speeds, we should not expect motions in the jet and counterjet to be correlated, whereas if the symmetric outflow is due to a common explosive origin the kinematics of each side might show similar properties. In at least NGC 1052 and Cygnus A we can make a one-to-one association between features on the two sides, supporting the idea that they reflect the real jet flow, although in M87 no such association can be made. We note that in all of these sources, the intrinsic flow speeds are only mildly relativistic, and the spatial scales are much smaller than the more distant objects; thus we cannot make any inferences from this argument about the nature of the apparent superluminal speeds that are observed in the more powerful quasars.

\subsection{Highly Compact Jets}

We have also identified three extremely core-dominated sources $(0235+164,1324+224,1741-038)$ which had very little discernible mas-scale jet structure at any epoch. This could either be because their jets are atypically weak, or they may possess a highly beamed core which swamps the jet emission in our limited dynamic range images. It is also possible that they may be experiencing a period of inactivity in which new jet components are not being ejected. The latter was the case for $1308+326$, which had very little dis- cernible jet structure prior to 1997, and now has a pronounced mas-scale jet.

With the exception of $0235+164$, none of the three sources show any extended radio structure in deep VLA images (Ulvestad et al. 1981; Perley et al. 1982; Cooper et al. 2007). They are therefore well suited as calibration sources for flux density and absolute polarization position angle scaling when simultaneous single-dish/VLA and VLBA data are available.

\subsection{Stable Jets}

VLBI sources that maintain a stable mas-scale structure for long periods with well-defined centroids are very useful in geodetic applications such as the definition of the International Celestial Reference Frame (Feissel-Vernier 2003). Our long-term VLBA study has indicated, however, that such sources are exceedingly rare. We have identified only two sources in the MOJAVE sample whose components have maximum apparent speeds smaller than $10 \mu \mathrm{as} \mathrm{y}^{-1}$. Both of these are at high redshift. Unlike the compact sources discussed in Section 3.7 which may be in temporarily quiescent states, the decade-long stability of these sources suggests that they have low intrinsic expansion velocities and/or high jet viewing angles. However, we cannot exclude the possibility that faster changes of the jet structure of these quasars might occur in the future.

$0552+398$ (DA 193). This AGN $(z=2.363)$ has frequently been classified as a gigahertz-peaked spectrum (GPS) source in the literature. However, it displays considerable flux density variability above its turnover frequency (Torniainen et al. 2005), and has persistent linear polarization on mas scales that is uncharacteristic of the GPS class. We were able to fit all of the $2 \mathrm{~cm}$ MOJAVE VLBA epochs between 1997 and 2007 with a two-component Gaussian model, in which the separation is barely increasing at a rate of $4 \pm 1 \mu$ as $\mathrm{y}^{-1}(0.36 \pm 0.09 c)$.

$0642+449$. This high redshift $(z=3.396)$ quasar has also been previously classified as a GPS, yet Torniainen et al. (2005) found its flux density variability to be too high for this class. During our 12 year monitoring interval we have found a single robust jet component whose core separation is increasing at only $7 \pm 1 \mu$ as y $^{-1}(0.76 \pm 0.11 c)$.

\section{OVERALL KINEMATICS OF THE MOJAVE JET SAMPLE}

\subsection{Distribution of Angular Jet Speeds}

The robust jet features in our sample span a wide range of apparent speed, measured in both angular units and in units of the speed of light. The distribution of angular speeds (Figure 6) provides useful information for the planning of kinematic monitoring programs (e.g., TANAMI: Kadler et al. 2007), since it indicates how often bright blazar jets should typically be observed to accurately track their motions. The distribution is roughly log-normal, with a median of 0.22 mas $^{-1}$. The feature with the largest measured proper motion is in the jet of the nearby radio galaxy $0430+052$ (3C 120) and is moving at $2.46 \pm 0.074$ mas $^{-1}$. In the MOJAVE survey, we have attempted to observe sources such that individual jet features move less than a beamwidth between observations. This facilitates the identification of features across successive epochs. Cadences range from one observation per month for the fastest sources to once every two to three years for the slowest sources, with a median of once every 11 months (Paper V). 


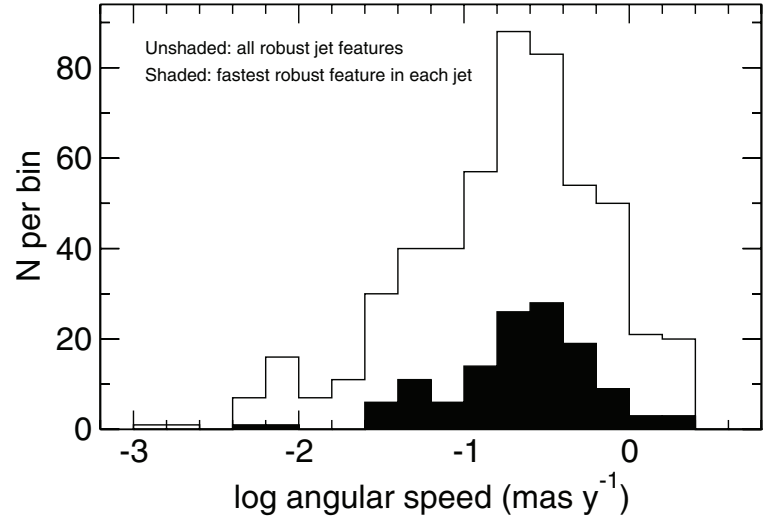

Figure 6. Angular speed distribution for all 526 robust jet features in the MOJAVE AGN sample. The shaded histogram represents the distribution of the fastest robust feature in each jet.

\subsection{Maximum Jet Speeds}

A primary goal of the MOJAVE survey is to measure the distribution of jet speeds in our flux-density-limited AGN sample, in order to investigate the overall speed distribution and beaming properties of the blazar parent population. Such information is needed for studies of AGN LFs and blazar demographics (e.g., Cara \& Lister 2008). The issue is complicated somewhat by the possible presence of multiple pattern speeds in an individual jet, some of which may be moving at a different speed than the bulk flow (Section 3.5). We are therefore interested in the fastest speed in the jet measured over our approximately decade-long monitoring period. For the purposes of constructing a maximum speed statistic for each source, we considered all robust components in Table 2 with angular speed measurements above the $3 \sigma$ level. This method addressed some isolated cases such as $2201+171$, where a diffuse component with large positional uncertainties gave a large (and poorly determined) speed measurement that dominated the statistic for the source. In the case of $0422+004$, which had only a single robust component, we adopted its speed as the maximum speed, despite its measured value being slightly below $3 \sigma$.

\subsubsection{Distribution of Maximum Jet Speeds}

In Figure 7, we plot the distribution of jet speeds for all robust features in the sample. The shaded histogram represents the distribution of maximum speed. The fall-off toward high values is consistent with previous large VLBI surveys carried out at longer observing wavelengths (Britzen et al. 2007; Piner et al. 2007). However, a major difference in the MOJAVE distribution is that it is peaked at a moderately high value $\left(\beta_{\text {app }} \simeq 10\right)$, whereas the earlier surveys were peaked at values $<4 c$. The selection criteria of all three surveys are similar, and contain many overlapping objects. The MOJAVE survey does have higher angular resolution and excellent temporal coverage, however, which may facilitate the identification of fast-moving components that would otherwise be missed. Indeed, our fastest identified jet component (component ID $=3$ in the quasar $\left.0805-077^{14}\right)$ at $50.6 \pm 2.1 c$ is significantly faster than any previously identified in cm-wavelength VLBA surveys, and is comparable to some of the fastest components identified in a

\footnotetext{
14 The redshift of $0805-077(z=1.817)$ was originally measured by White et al. (1988), on the basis of several broad emission lines in the optical spectrum, and was later confirmed at the Nordic Optical Telescope by T. Pursimo (2009, private communication).
}

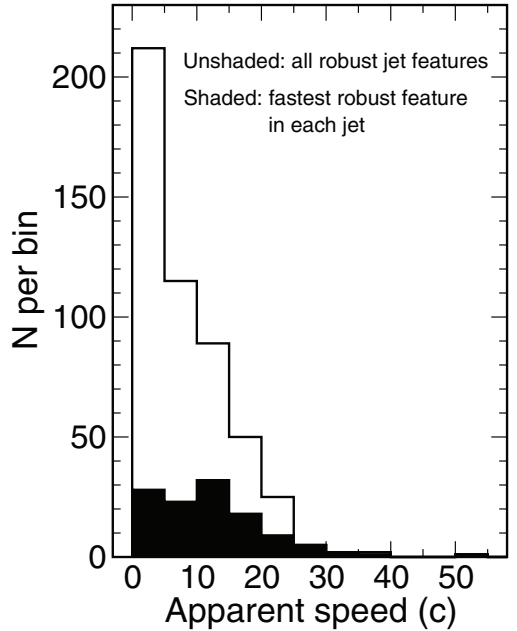

Figure 7. Distribution of apparent speed for 502 robust jet features in MOJAVE AGN with measured redshifts. The shaded histogram represents the distribution of the fastest robust feature in each jet.

small sample of 15 blazar jets observed monthly with the VLBA at $7 \mathrm{~mm}$ by Jorstad et al. (2005). The median measured speed in the latter study was also $\sim 10 c$, which suggests that high angular resolution is essential for determining accurate jet speeds in blazar proper motion surveys. Data from the Fermi Observatory have shown that the extremely fast MOJAVE blazars, including 0805-077 (Ciprini 2009a, 2009b), show a higher tendency to emit high-energy $\gamma$-rays, likely as a result of their high Doppler boosting factors (Lister et al. 2009; Kovalev et al. 2009).

Other studies (Vermeulen \& Cohen 1994; Lister \& Marscher 1997) have investigated how the distribution of fastest apparent speeds in an AGN sample selected on the basis of beamed jet emission reflects the overall distribution of Lorentz factors $(\Gamma)$ in the parent population. First, the maximum observed apparent speed sets an approximate value for $\Gamma_{\max }$, since $\Gamma>\left(\beta_{\mathrm{app}}^{2}+1\right)^{0.5}$, and in a large parent population, some of the jets with $\Gamma=\Gamma_{\max }$ will have their observed flux densities Doppler boosted by very large factors by virtue of their near end-on orientations. For reasonable choices of the parent LF, most of these sources will be selected in a flux-density-limited survey, regardless of their redshift. Second, the shape of the distribution in Figure 7 rules out a single-valued $\Gamma$ distribution, since in that case the apparent speed distribution would be expected to peak at $\beta_{\text {app }} \simeq \Gamma$, and drop off sharply for smaller values of $\beta_{\text {app }}$ (Vermeulen \& Cohen 1994). The tapering of the speed distribution at higher values strongly suggests a power-law Lorentz factor distribution, which we use in Monte Carlo beaming simulations described in Section 4.2.3.

\subsubsection{Maximum Speed Versus Redshift}

In Figure 8, we plot maximum speed against redshift for the full sample. The data points are approximately evenly scattered for redshifts greater than $\sim 0.6$, whereas at lower redshifts, there is an apparent upper envelope, indicating that the maximum superluminal speed increases with redshift. The dashed curve represents a constant angular velocity of 2.5 mas y $^{-1}$, which corresponds to the largest angular speed measured in the survey. Since this curve lies well above the data points past $z=0.1$, we conclude that the upper envelope is not the result of survey measurement bias.

The envelope instead likely arises because of the combined effects of relativistic beaming and the sample's lower flux 


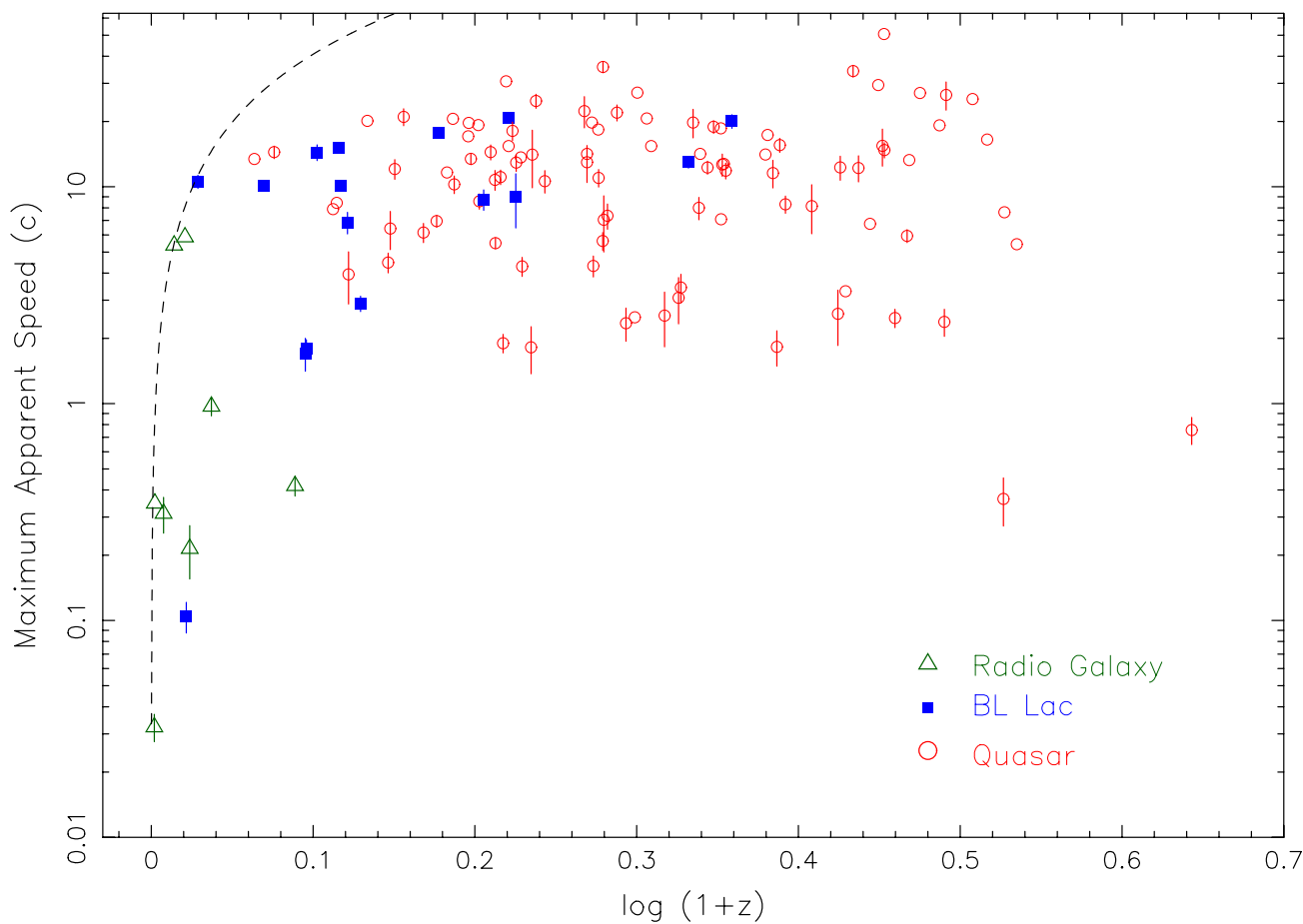

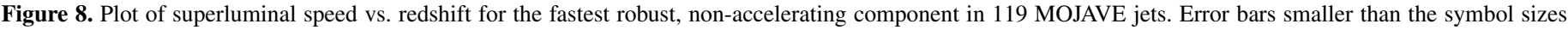

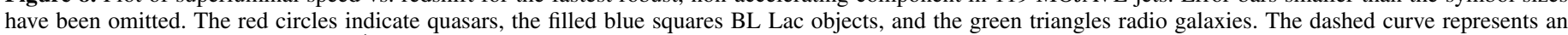
angular apparent speed of 2.5 mas $\mathrm{y}^{-1}$

(A color version of this figure is available in the online journal.)

density cutoff. In the MOJAVE survey, the minimum detectable luminosity rises sharply with redshift, creating a classical Malmquist bias (see Figure 1 of Cara \& Lister 2008). The highredshift sources therefore have higher apparent luminosities, which they achieve primarily via Doppler boosting. Our Monte Carlo simulations (Section 4.2.3) suggest that these jets have some of the highest Doppler boosting factors in the parent population (i.e., $\sim 2 \Gamma_{\max }$ ), and therefore can have apparent speeds up to $\beta_{\text {app }}=\Gamma_{\max }$. At lower redshifts (below $z \simeq 0.6$ ), beaming is not as important as a factor in determining whether a jet makes it into the sample, and the available co-moving volume is much smaller. This makes it far less probable that highly superluminal jets will be seen at low redshifts. The transition at which this occurs corresponds to a redshift $z_{o}$ where the survey luminosity limit roughly coincides with a flattening in the beamed LF. Lister (2003) showed that the latter occurs at $L_{1} \delta_{\max }^{p}$, where $L_{1}$ is the low-luminosity cutoff of the intrinsic LF, $\delta_{\max }$ is the maximum Doppler boosting factor, and $p$ is the boosting index. For a flat-spectrum jet with a typical boosting index of $p=$ $2, \delta_{\max }=2 \beta_{\text {app, } \max }=100$, and $L_{1}=1.2 \times 10^{23} \mathrm{~W} \mathrm{~Hz}^{-1}$, which corresponds to the faintest source in the survey $(1228+126$ $=$ M87), the transition occurs at $z_{0} \simeq 0.55$, in approximate agreement with Figure 8.

\subsubsection{Maximum Speed Versus Apparent Radio Luminosity}

In an earlier paper using preliminary MOJAVE data, Cohen et al. (2007) reported an upper envelope in a plot of maximum jet speed versus VLBA luminosity. In Figure 9, we present a slightly different version of this plot, where we have instead plotted the logarithm of the jet speed in order to alleviate crowding close to the $x$-axis. In calculating the luminosities, we have used the median total $2 \mathrm{~cm}$ VLBA flux density measured during the period 1994.0-2004.0 and have $k$-corrected the luminosities assuming a flat spectral index $(\alpha=0)$.

The dotted curve represents a jet with $\mu=2.5$ mas $^{-1}$, flux density of $1.5 \mathrm{Jy}$, and flat spectral index, plotted for a range of redshifts. These values correspond to the maximum angular speed measurement and the flux density limit of the survey, respectively. The slope of this curve is roughly $\sim 0.5$ in this $\log -\log$ plot, since the apparent velocity depends linearly on luminosity distance, whereas observed luminosity varies as luminosity distance squared (the $k$-correction causes a slight curvature at high $z$ ). As in the case of the maximum speed versus redshift plot (Figure 8), the empty region between this curve and the data points indicates that the envelope is not the result of observational measurement limits.

Cohen et al. (2007) pointed out that the shape of the envelope was very similar to the locus of points created by a single jet oriented over a range of different viewing angles. The free parameters involved in fitting this "aspect curve" to the envelope are the jet's unbeamed luminosity (which determines its horizontal shift), its Lorentz factor (which determines its shape and vertical extent), and the Doppler boost index $p$, (which influences both the curve's shape and its slope at low apparent luminosities).

For reasonably high values of the Lorentz factor, we can derive the approximate slope of the left-hand side of the aspect curve, where $2 / \Gamma \lesssim \delta \ll \Gamma$ (i.e., $\theta \gg \sin ^{-1}(1 / \Gamma)$ ):

$$
\beta_{\text {app }}^{2}=2 \delta \Gamma-\delta^{2}-1
$$

$$
\simeq 2 \delta \Gamma \text {. }
$$

We also have

$$
L=C_{1} \delta^{p},
$$




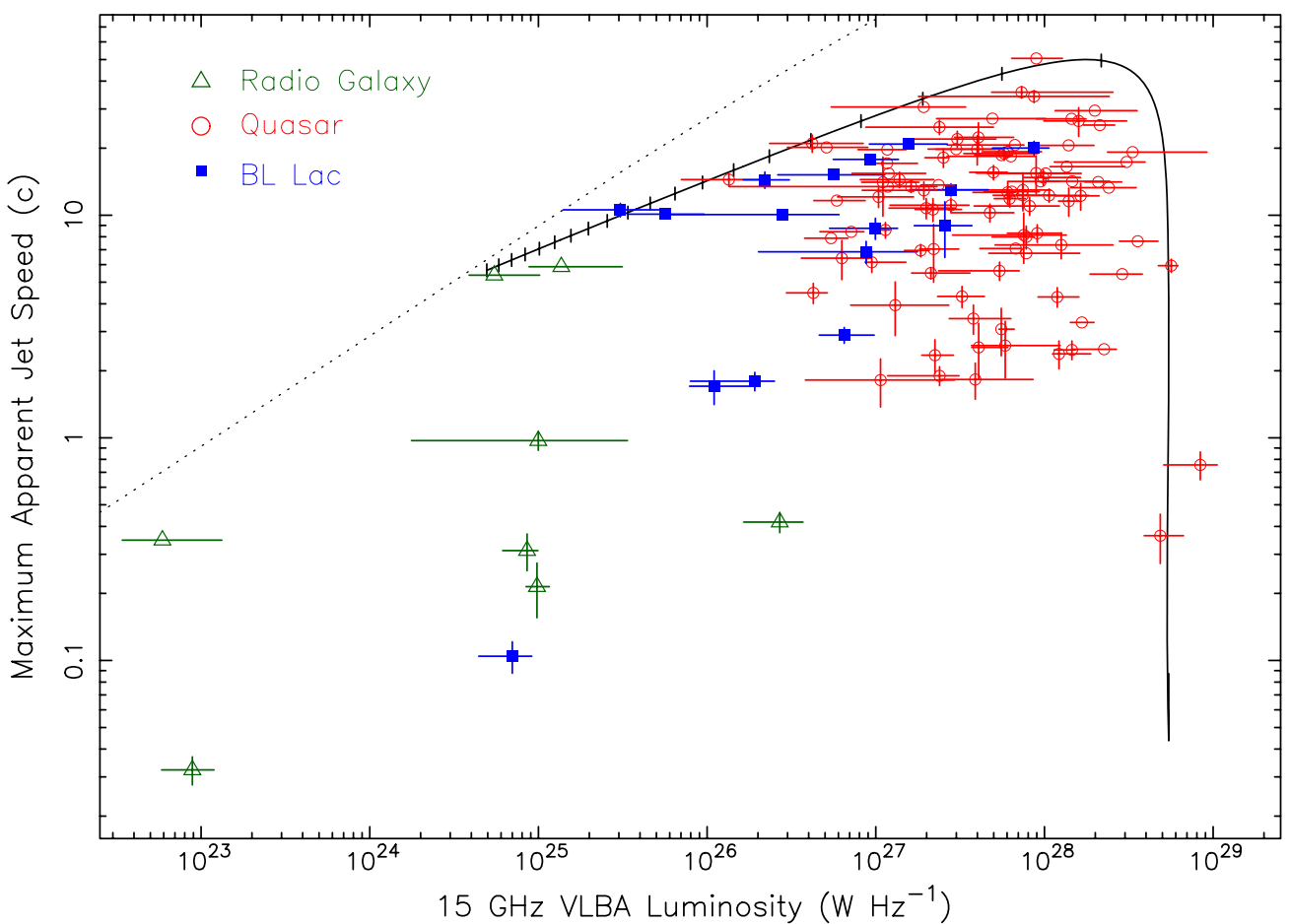

Figure 9. Plot of maximum jet speed vs. $2 \mathrm{~cm}$ VLBA luminosity for 119 jets in the MOJAVE survey. The red circles indicate quasars, the filled blue squares BL Lac objects, and the green triangles radio galaxies. Vertical error bars smaller than the symbol sizes have been omitted. The horizontal error bars indicate the range of luminosity of each source during the period 1994.0-2004.0, and the symbols correspond to the median values. The dotted curve corresponds to a jet with an apparent speed of 2.5 mas $^{-1}$ and a flux density of $1.5 \mathrm{Jy}$, plotted over a range of redshifts. The solid curve represents a jet at viewing angles ranging from $5 \times 10^{-4}$ deg to $20^{\circ}$, with Lorentz factor $=50$ and an intrinsic unbeamed luminosity of $3 \times 10^{25} \mathrm{~W} \mathrm{~Hz}^{-1}$, Doppler boosted by a factor $\delta^{1.63}$. Ticks are plotted along the solid curve at $1^{\circ}$ intervals, starting at a viewing angle of $1^{\circ}$ and increasing toward the left.

(A color version of this figure is available in the online journal.)

where $C_{1}$ is a constant. Combining Equations (4) and (5) yields

$$
\beta_{\text {app }}=\sqrt{2 \Gamma}\left(L / C_{1}\right)^{(1 / 2 p)},
$$

thus the slope in the $\log \beta_{\text {app }}$ versus $\log L$ plot is $0.5 p^{-1}$.

The solid curve in Figure 9 represents a jet with the Lorentz factor $\Gamma=50$ and an intrinsic unbeamed luminosity of $3 \times$ $10^{25} \mathrm{~W} \mathrm{~Hz}^{-1}$, viewed at angles ranging from $5 \times 10^{-4}$ to $20^{\circ}$, and Doppler boosted by a factor $\delta^{1.63}$. The tick marks are located at $1^{\circ}$ intervals moving from right to left, beginning at $1^{\circ}$ from the line of sight. The aspect curve provides a reasonably good fit to the envelope, although there is considerable freedom in the fitting parameters. We determined the boosting index by performing a least-squares fit to the 10 data points between $10^{24.7} \mathrm{~W} \mathrm{~Hz}^{-1}<$ $L<10^{28} \mathrm{~W} \mathrm{~Hz}^{-1}$ that lie along the upper envelope. This yielded a slope of $0.307 \pm 0.015$, which corresponds to $p=1.63 \pm 0.08$. This Doppler boost is somewhat lower than that expected from a continuous flat-spectrum jet $(p=2-\alpha$, where $\alpha=0$ ).

Although the aspect curve appears to provide a good fit to the upper envelope, we find little justification for it in the framework of the relativistic beaming model. One interpretation would require that every jet located on the upper left edge of the envelope have a Lorentz factor of 50 and an identical unbeamed luminosity. The lowest apparent luminosity jets in this subgroup would then have viewing angles of $10^{\circ}-20^{\circ}$, and their Doppler factors would be on the order of unity. However, since we are dealing with a flux-density-limited beamed sample, there is a strong preference for high-Doppler factor jets. At a given observed luminosity, e.g., $10^{26} \mathrm{~W} \mathrm{~Hz}^{-1}$, there is a much higher probability of having a jet with lower intrinsic luminosity and moderately high Doppler factor, in part because of the steepness of the parent luminosity function, and also because of the rarity of ultra-high Lorentz factor jets in the parent population (see Section 4.2.1). Thus, there is no a priori reason to expect a sharp upper envelope bounded by a curve with $\Gamma=\Gamma_{\max }$. Another possible explanation for the envelope, explored by Lister \& Marscher (1997) and Cohen et al. (2007), is that there is a correlation between intrinsic jet power and Lorentz factor, such that a jet with a particular $\Gamma$ must have a certain minimum unbeamed luminosity $L(\Gamma)$.

We have attempted to investigate these possibilities by performing Monte Carlo simulations of a beamed parent population, from which we select randomly generated jets above the MOJAVE flux density threshold. The intrinsic jet luminosities are generated assuming a power-law pure-density evolution LF with slope -2.65 and redshifts between 0.04 and 4 , as described by Cara \& Lister (2008). Because of the small number of lowluminosity jets in the MOJAVE sample, these parameters are chosen to optimize the fit to the quasar population. The jets are assigned random orientations, and their Lorentz factors are drawn from a power-law distribution with slope $=-1.5$ and $3<\Gamma<50$. The latter provides a reasonable fit to the overall distribution of maximum jet speeds (Section 4.2.1). Each jet is beamed using a Doppler boost index of $p=1.63$. The simulation is provided a random number seed, and jets are generated until a flux-density-limited sample of comparable size to the MOJAVE survey is obtained. With the choice of parameters above, and $10^{24} \mathrm{~W} \mathrm{~Hz}^{-1}<L_{\text {intrinsic }}<10^{27.6} \mathrm{~W} \mathrm{~Hz}^{-1}$, this typically required $\sim 20$ million parent jets.

In Figure 10, we show the results of two simulations using these parameters, albeit with different random number seeds. 

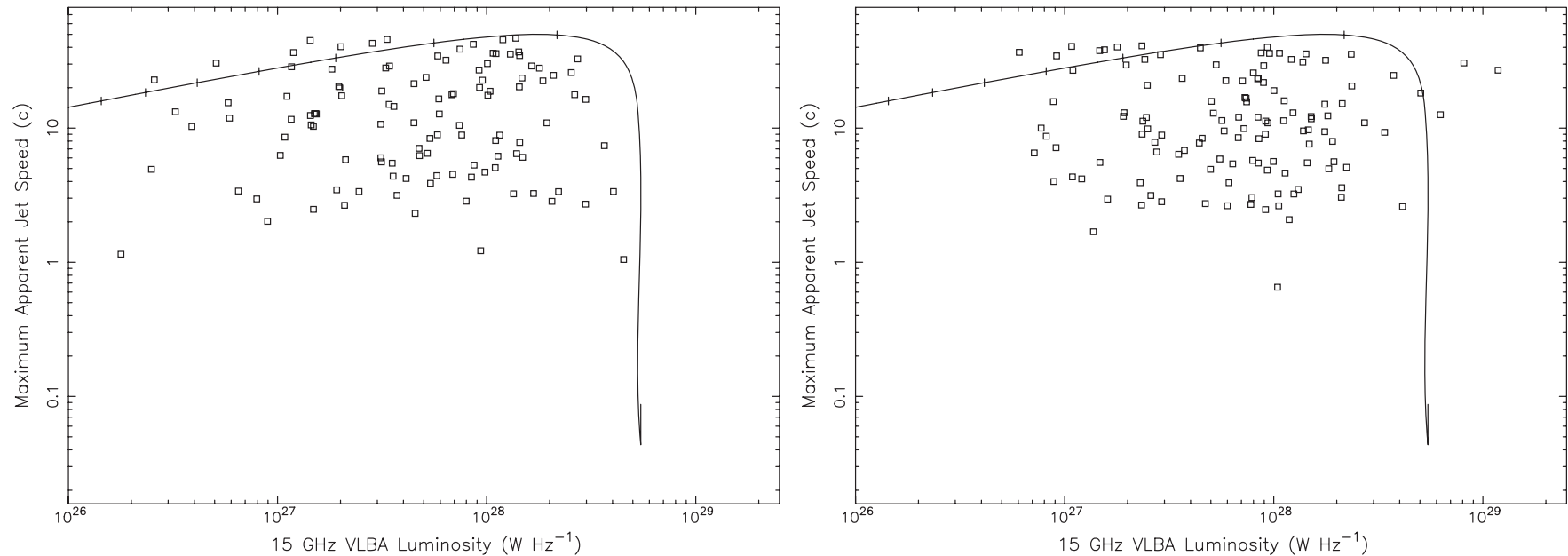

Figure 10. Left panel: plot of apparent speed vs. observed luminosity for 135 simulated jets (see Section 4.2.3). The solid curve is identical to that in Figure 9; however, the plotted $x$-axis ranges of the panels are different. Right panel: identical simulation to the left-hand panel, but with a different random number seed.

The fact that one of the simulations appears to reproduce the envelope, while the other does not, reflects the statistical fluctuations that are inherent in selecting a biased subset of sources from a very large parent population. The apparent flux density of any particular jet is determined by the joint probability density of four variables, namely redshift, intrinsic luminosity, viewing angle, and Lorentz factor. Thus, there are multiple ways in which a jet can make it into a beamed sample, such as having a low redshift, or a high Doppler factor, or both. Since the flux density limit of the MOJAVE survey is shallow, we are likely situated too far out in the statistical tail to fully constrain particular aspects of the parent population, such as an intrinsic relation between speed and luminosity. In order to address this issue, we are obtaining speed information on an extended sample of AGN jets that includes low-luminosity radio galaxies and new $\gamma$-ray blazars detected by the Fermi Observatory.

\section{SUMMARY}

We have investigated the parsec-scale kinematic properties of 135 jets in the complete MOJAVE flux-density-limited survey of compact, radio-selected AGNs. The observational data set consists of 2424 VLBA images at $2 \mathrm{~cm}$ from our program and the VLBA archive between 1994 August 31 and 2007 September 6, and represents a significant improvement over previous multi-epoch AGN surveys in terms of statistical completeness, temporal coverage, and overall data quality.

Our main results can be summarized as follows:

1. By modeling individual bright jet features with Gaussian components, we were able to robustly identify and track a total of 526 moving features in 127 AGN jets. Three AGN $(0235+164,1324+224,1741-038)$ had very little discernible mas-scale jet structure at any epoch, while five others displayed too much scatter to reliably crossidentify their features across multiple epochs. We have also identified two bright AGN $(0552+398$ and $0642+449)$ with extremely stable mas-scale structures that have changed by less than $10 \mu$ as $\mathrm{y}^{-1}$ over a 12 year interval.

2. We fit a simple linear model to the positions of each component over time in order to determine its speed and direction with respect to the (presumed stationary) core feature located near the base of the jet. Approximately one third of the components had velocity vectors which did not extrapolate back to the core feature. A more detailed analysis presented elsewhere (Homan et al. 2009) links these "non-radial" motions to accelerations perpendicular to the velocity vector.

3. The jet features show an overwhelming tendency to display outward motions, away from the core $(98.5 \%$ of all components). Our analysis revealed only eight inward-moving components, and in only one case (ID = 2 in $2351+456$ ) is the motion highly superluminal. VLBA data obtained since 2007 on the latter feature has indicated that its inward motion has stopped, suggesting that it may be a case where the jet has crossed our line of sight.

4. Features within an individual jet tend to have a modestly narrow distribution of apparent speed (three times smaller than the overall speed dispersion in the sample), supporting the idea that they are connected with the intrinsic flow. We identified only 31 features whose motions were slow and significantly smaller than others in the same jet. These "LPS" features are more prevalent in the BL Lac objects (10 of 22) than in the quasars (15 of 86), and are rarely found at de-projected distances greater than $50 \mathrm{pc}$ downstream of the core. They may represent strong, stable re-collimation shocks of the type commonly seen in numerical jet simulations.

5. We have analyzed the kinematics of five MOJAVE sources that have jets of similar brightness located on either side of the central AGN. We find that at least two (NGC 1052 and Cygnus A) of these two-sided jets display a close similarity in the ejection epochs and apparent speeds of their jet and counterjet, providing additional evidence that the moving features are related to the flow.

6. Nearly $60 \%$ of the 526 robust components in the sample had at least 10 epochs of measurement; for these we also performed a two-dimensional fit to determine their velocities and accelerations. Approximately one third of these display significant accelerations at the $3 \sigma$ level, indicating that changes in speed and/or direction are a common feature of AGN jets.

7. We have compiled a maximum speed statistic for each jet, which is equal to the fastest robust feature observed in the source. The distribution of these maximum speeds is peaked at $\beta_{\text {app }} \simeq 10$, and falls off sharply toward higher values. This is indicative of a parent population that has a distribution of bulk Lorentz factors that is not peaked at high values. The fastest jet in our sample (0805-077) 
contains a component moving at $50.6 \pm 2.1 c$, which is likely representative of the upper end of the AGN jet Lorentz factor distribution.

8. The maximum jet speed increases with redshift out to $z \simeq 1$, which reflects a selection bias toward higher Lorentz factor jets at higher redshifts. At closer distances, the jets do not require as high beaming factors to meet the flux density selection criteria, and the probability finding a highluminosity, high-Lorentz factor jet is much lower because of the smaller co-moving volume available.

9. We have re-examined the upper envelope in the $\beta_{\text {app }}$ versus apparent luminosity plane first reported by Cohen et al. (2007) using our new data set. The envelope is not the result of any observational measurement bias, and is very similar in shape to an "aspect curve" on the plane traced by a single high-Lorentz factor jet as it is rotated through different viewing angles. The statistical probability of having this envelope, however, is low, in terms of the standard beaming model, which we have confirmed using Monte Carlo simulations with multiple random number seeds. It is possible instead that the envelope arises because of an intrinsic relation between jet speed and luminosity in the parent population, although deeper samples will likely be needed to fully investigate this scenario.

The authors acknowledge the contributions of additional members of the MOJAVE team: Hugh Aller, Margo Aller, Ivan Agudo, Andrei Lobanov, Alexander Pushkarev, Kirill Sokolovsky, and Rene Vermeulen. Several students also contributed to this work: Christian Fromm at MPIfR, and Amy Lankey, Kevin O'Brien, Ben Mohlie, and Nick Mellott at Purdue University. M.L.L. has been supported under NSF grants AST0406923 \& AST-0807860, NASA-Fermi grant NNX08AV67G, and a grant from the Purdue Research Foundation. D.C.H. is supported by NSF grant AST-0707693. T.S. has been supported in part by the Academy of Finland grant 120516. M.K. has been supported in part by an appointment to the NASA Postdoctoral Program at the Goddard Space Flight Center, administered by Oak Ridge Associated Universities through a contract with NASA. Part of this work was done by Y.Y.K. and T.S. during their Alexander von Humboldt fellowships at the MPIfR. The National Radio Astronomy Observatory is a facility of the National Science Foundation operated under cooperative agreement by Associated Universities, Inc. This research has made use of NASA's Astrophysics Data System, and the NASA/IPAC Extragalactic Database (NED). The latter is operated by the Jet Propulsion Laboratory, California Institute of Technology, under contract with the National Aeronautics and Space Administration.

Facilities: VLBA ()

\section{REFERENCES}

Abdo, A. A., et al. 2009, ApJ, 699, 31

Acciari, V. A., et al. 2009, Science, 325, 444

Alberdi, A., Gómez, J. L., Marcaide, J. M., Marscher, A. P., \& Pérez-Torres,

M. A. 2000, A\&A, 361, 529

Alberdi, A., et al. 1997, A\&A, 327, 513

Bach, U., Kadler, M., Krichbaum, T. P., Middelberg, E., Alef, W., Witzel, A., \& Zensus, J. A. 2005, in ASP Conf. Proc. 340, Future Directions in High Resolution Astronomy, ed. J. Romney \& M. Reid (San Francisco, CA: ASP), 30

Becker, P. A., Das, S., \& Le, T. 2008, ApJ, 677, L93

Britzen, S., et al. 2005, A\&A, 444, 443

Britzen, S., et al. 2007, A\&A, 472, 763
Britzen, S., et al. 2008, A\&A, 484, 119

Cara, M., \& Lister, M. L. 2008, ApJ, 686, 148

Ciprini, S. 2009a, Atl, 2048, 1

Ciprini, S. 2009b, Atl, 2136, 1

Cohen, M. H., Lister, M. L., Homan, D. C., Kadler, M., Kellermann, K. I., Kovalev, Y. Y., \& Vermeulen, R. C. 2007, ApJ, 658, 232

Cooper, N. J., Lister, M. L., \& Kochanczyk, M. D. 2007, ApJS, 171, 376

D’Arcangelo, F. D., et al. 2007, ApJ, 659, L107

Dhawan, V., Kellermann, K. I., \& Romney, J. D. 1998, ApJ, 498, L111

Falomo, R. 1996, MNRAS, 283, 241

Feissel-Vernier, M. 2003, A\&A, 403, 105

Gómez, J. L., Martí, J. M. A., Marscher, A. P., Ibáñez, J. M. A., \& Alberdi, A. 1997, ApJ, 482, L33

Gómez, J. L., Martí, J. M. A., Marscher, A. P., Ibáñez, J. M. A., \& Marcaide, J. M. 1995, ApJ, 449, L19

Gong, B. 2008, MNRAS, 389, 315

Homan, D. C., Lister, M. L., Kellermann, K. I., Cohen, M. H., Ros, E., Zensus, J. A., Kadler, M., \& Vermeulen, R. C. 2003, ApJ, 589, L9

Homan, D. C., Ojha, R., Wardle, J. F. C., Roberts, D. H., Aller, M. F., Aller, H. D., \& Hughes, P. A. 2001, ApJ, 549, 840

Homan, D. C., Ojha, R., Wardle, J. F. C., Roberts, D. H., Aller, M. F., Aller, H. D., \& Hughes, P. A. 2002, ApJ, 568, 99

Homan, D. C., et al. 2006, ApJ, 642, L115

Homan, D. C., et al. 2009, ApJ, in press

Istomin, Y. N., \& Pariev, V. I. 1996, MNRAS, 281, 1

Jorstad, S. G., Marscher, A. P., Mattox, J. R., Aller, M. F., Aller, H. D., Wehrle, A. E., \& Bloom, S. D. 2001, ApJ, 556, 738

Jorstad, S. G., et al. 2005, AJ, 130, 1418

Kadler, M., Kerp, J., Ros, E., Falcke, H., Pogge, R. W., \& Zensus, J. A. 2004, A\&A, 420, 467

Kadler, M., Ros, E., Lobanov, A. P., Falcke, H., \& Zensus, J. A. 2004, A\&A, 426, 481

Kadler, M., et al. 2008, ApJ, 680, 867

Kellermann, K. I., et al. 2004, ApJ, 609, 539

Kovalev, Y. Y., Lister, M. L., Homan, D. C., \& Kellermann, K. I. 2007, ApJ, 668, L27

Kovalev, Y. Y., Lobanov, A. P., Pushkarev, A. B., \& Zensus, J. A. 2008, A\&A, 483,759

Kovalev, Y. Y., Nizhelsky, N. A., Kovalev, Y. A., Berlin, A. B., Zhekanis, G. V., Mingaliev, M. G., \& Bogdantsov, A. V. 1999, A\&AS, 139, 545

Kovalev, Y. Y., et al. 2005, AJ, 130, 2473

Kovalev, Y. Y., et al. 2009, ApJ, 696, L17

Lister, M. L. 2001, ApJ, 562, 208

Lister, M. L., \& Homan, D. C. 2005, AJ, 130, 1389

Lister, M. L., Homan, D. C., Kadler, M., Kellermann, K. I., Kovalev, Y. Y., Ros, E., Savolainen, T., \& Zensus, J. A. 2009, ApJ, 696, L22

Lister, M. L., Kellermann, K. I., Vermeulen, R. C., Cohen, M. H., Zensus, J. A., \& Ros, E. 2003, ApJ, 584, 135

Lister, M. L., \& Marscher, A. P. 1997, ApJ, 476, 572

Lister, M. L., Tingay, S. J., Murphy, D. W., Piner, B. G., Jones, D. L., \& Preston, R. A. 2001, ApJ, 554, 948

Lister, M. L., \& Smith, P. S. 2000, ApJ, 541, 66

Lister, M. L., et al. 2009, AJ, 137, 3718 (Paper V)

Lobanov, A. P. 1998, A\&A, 330, 79

Ly, C., Walker, R. C., \& Junor, W. 2007, ApJ, 660, 200

Mandal, S., \& Chakrabarti, S. K. 2008, ApJ, 689, L17

Marscher, A. P., Jorstad, S. G., Mattox, J. R., \& Wehrle, A. E. 2002, ApJ, 577, 85

Marscher, A. P., Zhang, Y. F., Shaffer, D. B., Aller, H. D., \& Aller, M. F. 1991, ApJ, 371, 491

Marscher, A. P., et al. 2008, Nature, 452, 966

McIntosh, D. H., Rieke, M. J., Rix, H.-W., Foltz, C. B., \& Weymann, R. J. 1999, ApJ, 514, 40

Moellenbrock, G. 1998, PhD thesis, Brandeis Univ.

Perley, R. A., Fomalont, E. B., \& Johnston, K. J. 1982, ApJ, 255, L93

Perlman, E. S., Stocke, J. T., Carilli, C. L., Sugiho, M., Tashiro, M., Madejski, G., Wang, Q. D., \& Conway, J. 2002, AJ, 124, 2401

Perucho, M., \& Martí, J. M. 2007, MNRAS, 382, 526

Piner, B. G., Mahmud, M., Fey, A. L., \& Gospodinova, K. 2007, AJ, 133, 2357

Press, W. H., et al. 2007, in Numerical Recipes, 3rd ed.: The Art of Scientific Computing (Cambridge: Cambridge Univ. Press), 776

Shepherd, M. C. 1997, in ASP Conf. Ser. 125, Astronomical Data Analysis Software and Systems VI, ed. G. Hunt \& H. E. Payne (San Francisco, CA: ASP), 77

Taylor, G. B., Vermeulen, R. C., Readhead, A. C. S., Pearson, T. J., Henstock, D. R., \& Wilkinson, P. N. 1996, ApJS, 107, 37 
Torniainen, I., Tornikoski, M., Teräsranta, H., Aller, M. F., \& Aller, H. D. 2005, A\&A, 435, 839

Ulvestad, J., Johnston, K., Perley, R., \& Fomalont, E. 1981, AJ, 86, 1010

Vermeulen, R. C., \& Cohen, M. H. 1994, ApJ, 430, 467

Vermeulen, R. C., Ros, E., Kellermann, K. I., Cohen, M. H., Zensus, J. A., \& van Langevelde, H. J. 2003, A\&A, 401, 113
Véron-Cetty, M.-P., \& Véron, P. 2006, A\&A, 455, 773

Walker, R. C., Dhawan, V., Romney, J. D., Kellermann, K. I., \& Vermeulen, R. C. 2000, ApJ, 530, 233

White, G. L., Jauncey, D. L., Wright, A. E., Batty, M. J., Savage, A., Peterson, B. A., \& Gulkis, S. 1988, ApJ, 327, 561

Wrobel, J. M. 1984, ApJ, 284, 531 